\title{
miRNA Transcriptome of Hypertrophic Skeletal Muscle with Overexpressed Myostatin Propeptide
}

\author{
Ruheena Javed, ${ }^{1}$ Lu Jing, ${ }^{1}$ Jinzeng Yang, ${ }^{2}$ Xinyun Li, ${ }^{1}$ Jianhua Cao, ${ }^{1}$ and Shuhong Zhao ${ }^{1}$ \\ ${ }^{1}$ Department of Animal Genetics \& Breeding, College of Animal Science and Veterinary Medicine, Huazhong Agricultural University, \\ Wuhan, Hubei Province 430070, China \\ ${ }^{2}$ Department of Human Nutrition, Food and Animal Sciences, University of Hawaii at Manoa, Honolulu, HI 96822, USA
}

Correspondence should be addressed to Shuhong Zhao; shzhao@mail.hzau.edu.cn

Received 21 April 2014; Revised 9 June 2014; Accepted 16 June 2014; Published 24 July 2014

Academic Editor: Qinghua Nie

Copyright (C) 2014 Ruheena Javed et al. This is an open access article distributed under the Creative Commons Attribution License, which permits unrestricted use, distribution, and reproduction in any medium, provided the original work is properly cited.

\begin{abstract}
MicroRNAs (miRNAs) play an imperative role in cell proliferation, differentiation, and cell metabolism through regulation of gene expression. Skeletal muscle hypertrophy that results from myostatin depression by its propeptide provides an interesting model to understand how miRNA transcriptome is involved in myostatin-based fiber hypertrophy. This study employed Solexa deep sequencing followed by Q-PCR methods to analyze miRNA transcriptome of skeletal muscle of myostatin propeptide transgenic mice in comparison with their littermate controls. A total of 461 mature known and 69 novel miRNAs were reported from this study. Fifty-seven miRNAs were expressed differentially between transgenic and littermate controls, of which most abundant miRNAs, miR-133a and 378a, were significantly differentially expressed. Expression profiling was validated on 8 known and 2 novel miRNAs. The miRNA targets prediction and pathway analysis showed that FST, SMAD3, TGFBR1, and AcvRla genes play a vital role in skeletal muscle hypertrophy in the myostatin propeptide transgenic mice. It is predicted that miR-101 targeted to TGFBR1 and SMAD3, miR-425 to TGFBR2 and FST, and miR-199a to AcvR2a and TGF- $\beta$ genes. In conclusion, the study offers initial miRNA profiling and methodology of miRNA targets prediction for myostatin-based hypertrophy. These differentially expressed miRNAs are proposed as candidate miRNAs for skeletal muscle hypertrophy.
\end{abstract}

\section{Introduction}

Skeletal muscle growth and maintenance are essential for animal and human health as $40-60 \%$ of the total body mass is composed of skeletal muscles, which provide structural support and enable the body to maintain posture, to control motor movements, and to store energy [1]. Skeletal muscle displays a remarkable plasticity with great capacity to alter its size, structure, and function in response to various stimuli. It therefore plays a vital role in the whole body metabolism. The muscle fibers are established early in life, and muscle growth and development are regulated by an interesting protein named myostatin, a cytokine synthesized in skeletal muscle cells. Myostatin (MSTN), also known as growth and differentiation factor-8 (GDF-8), is a member of the transforming growth factor- $\beta$ (TGF- $\beta$ ) superfamily and was identified by McPherron et al. [2]. Myostatin is expressed in skeletal muscle tissue where it functions to suppress myoblast proliferation and myofiber hypertrophy [3]. Genetic disruption of myostatin causes a dramatic increase in muscle mass. MSTN binds to activin receptor type IIB (ActRIIB) to exert its biological activity [4-6]. The binding of MSTN to its receptor leads to phosphorylation of transcription factors Smad2 and Smad3 along with subsequently forming a complex with Smad4, resulting in nuclear translocation of the Smad complex and consequent regulation of transcription of downstream target genes [7-9].

Myostatin mRNA is conserved across many mammalian species and massive muscle growth is observed in several livestock animal species with mutations in MSTN gene [10]. Previously, studies reported that MSTN-knockout mice show muscle fiber hyperplasia and hypertrophy in comparison with wild type mice [2]. Like other TGF- $\beta$ family members, myostatin is synthesized as a precursor protein, which 
undergoes two posttranslational cleavage events, generating an $\mathrm{N}$-terminal and a C-terminal peptides. The N-terminal peptide is referred to as myostatin propeptide while the $\mathrm{C}$ terminal peptide is the actual mature form of myostatin. Transgenic overexpression of myostatin propeptide cDNA in skeletal muscle increases animal growth and muscle mass $[11,12]$. Enhanced muscle mass phenotype in the propeptide transgenic mice primarily results from myofiber hypertrophy rather than myofiber hyperplasia. The size of fast-twitch, glycolytic muscle fiber at 9 weeks of age was increased by $60 \%$ compared with wild-type littermates [11]. By systemic comparisons of global mRNA expression in hypertrophic muscle of the transgenic mice using microarray and qRT-PCR techniques, the distinct expression patterns are identified which are comprised of enhanced expressions of myogenic regulatory factors and extracellular matrix components and differentially downregulated expressions of genes related to protein degradation and mitochondrial ATP synthesis [13, 14].

MicroRNAs are reported to act as negative regulators of target gene expression by recruiting silencing complexes to complementary sequence elements in target mRNAs. miRNAs are small 19-24 nucleotide (nt) RNAs that generally modulate gene expression through translational repression or by causing deadenylation and degradation of target mRNAs $[15,16]$. Bioinformatic studies have demonstrated that each miRNA has hundreds of target genes in animals, and up to $30 \%$ of all animal genes are miRNA targets [17-22]. Previous studies demonstrated that miRNAs play an essential role in skeletal muscle development by regulating gene expression. In skeletal muscle, miRNAs have been implicated in proliferation, differentiation, hypertrophy, regeneration, and disease [23]. They are functionally significant and potentially key regulators of gene expression during skeletal muscle development [24-29]. For example, deletion of a conditional Dicer allele in embryonic skeletal muscle results in perinatal lethality due to skeletal muscle hypoplasia [30]. The pivotal roles of three muscle-specific miRNAs, miR-1, miR133, and miR-206, in the regulation of myogenesis have been well documented $[17,31,32]$. A group of miRNAs, highly enriched in skeletal muscle (referred to as myomiRs), has recently been identified and includes miR-1, miR-133a, miR-133b, miR-206, miR-208, miR-208b, miR-486, and miR499 [33-37]. Several of these miRNAs are organized under bicistronic clusters on the same chromosome (i.e., miR-11/133a-2, miR-1-2/133a-1, and miR-206/133b) and are transcribed together [17, 38, 39]. Regulation of these myomiRs is controlled by key myogenic regulatory factors (MRFs), including myogenic differentiation $1(\mathrm{MyoD})$ and myogenin $[40,41]$ as well as myocyte enhancer factor 2 (MEF2) [42], serum response factor (SRF) [23], and myocardin-related transcription factor-A (MRTF-A) [33]. MyomiRs influence multiple facets of muscle development and function through regulation of the key myogenic genes [23, 40, 43]. Recent studies demonstrated that miR-29b, miR-133a, and 133b regulate myoblast proliferation and differentiation [38, 44], and miR-1 and miR-133 have been reported to regulate different aspects of skeletal muscle development in vitro and in vivo [23]. miR-1 promotes myocyte differentiation by repressing the expression of histone deacetylase 4 (HDAC4), a negative regulator of differentiation and a repressor of the MEF2 (myocyte enhancer factor-2) transcription factor [23].

MSTN gene also can regulate certain miRNAs expression through feedback autoregulation mechanism [45]. In Piedmontese cattle with mutated myostatin gene, MSTN gene was found to be targeted by miR-27b and miR-27b expression was correlated with hypertrophic phenotype of Piedmontese cattle [46]. A recent study further confirmed that MSTN regulates $\mathrm{miR}-27 \mathrm{a} / \mathrm{b}$ via SMAD3 in feedback autoregulation manner. Increased expression of $\mathrm{miR}-27 \mathrm{a} / \mathrm{b}$ was correlated with decreased expression of MSTN and vice versa both in vitro and in vivo mouse model. Loss of SMAD3 was associated with an increased level of MSTN [47].

Although the functional significances of miRNAs in controlling myogenesis have been documented and the majority of miRNAs are abundantly expressed, identifications of novel miRNAs that are expressed at low levels during skeletal muscle development have not been well defined. With robust approaches such as high-throughput deep sequencing technology, we can produce the whole miRNA transcriptomes [48]. In this study, we have attempted to identify candidate miRNAs in hypertrophic skeletal muscle with transgenic overexpressed myostatin propeptide by using transcriptome deep sequencing. Sequencing results have shown that a group of highly abundant known miRNAs were expressed in skeletal muscle. Using computational target prediction software we have identified differentially expressed miRNAs associated with muscle hypertrophy. An interaction network between miRNA and identified targets was constructed.

\section{Materials and Method}

2.1. Muscle Tissue Sample Collection. Myostatin propeptide transgenic mice were generated by standard microinjection techniques using B6SJL F1 females (Taconic, Germantown, NY) as zygote donors using the transgene MLC-pro construct [11]. The MLC-pro construct consists of myosin light chain (MLC) promoter and enhancer, myostatin cDNA from the 50 untranslated regions to the nucleotides encoding the propeptide cleavage site (RSRR), and SV40 PolyA tail signal sequence. Transgenic mice and wild-type littermate mice were obtained from offspring of MLC-pro transgenic mice mating with B6SJL F1. All procedures and animal care were in accordance with the institution guidelines and approved by the Institutional Animal Care and Use Committee of the University of Hawaii, Hawaii, USA. Myostatin propeptidetransgenic mice were generated by standard microinjection techniques, which has been previously described [9]. Male mice (hemizygous genotype for the transgene) from the high-expressing line were mated with B6SJL F1 wild-type females to produce offspring mice, which were used in this study. Mice were housed in cages; room temperature was maintained at $22^{\circ} \mathrm{C}$ and $12 \mathrm{~h}$ light/dark cycle. Mice were weaned at 4 weeks of age and given free access to a chow diet $(10 \% \mathrm{kcal}$ fat, ME3.85 kcal $/ \mathrm{g})$. Male mice at 4 months age were sacrificed for muscle tissue dissections and sampling after $8 \mathrm{hr}$ fasting. Gastrocnemius muscle samples 
TABLE 1: Number of miRNA reads from control and transgenic libraries.

\begin{tabular}{lccccc}
\hline Reads & CN148 & CN150 & TN126 & TN135 & TN329 \\
\hline Total reads & 11303077 & 9357529 & 9734519 & 10207035 & 21774919 \\
Clean reads & 10479962 & 8375356 & 9052728 & 9677365 & 20070380 \\
Qualified\% & $92.728 \%$ & $89.504 \%$ & $92.996 \%$ & $94.812 \%$ & $92.172 \%$ \\
Mapped reads & 536489 & 224550 & 177471 & 190093 & 429933 \\
Mapped\% & 5.119188 & 2.68108 & 1.960415 & 1.964305 & 2.142126856 \\
\hline
\end{tabular}

from both legs were immediately dissected from carcass, cleaned from fat, blood and quickly frozen in liquid nitrogen, and later stored at $-80^{\circ} \mathrm{C}$. Two wild-type littermate control samples, that is, CN148 and CN150, and three transgenic mouse samples, that is, TN126, TN135, and TN 329, were, respectively, collected from gastrocnemius muscle. Samples were immediately frozen in liquid nitrogen and stored at $-80^{\circ} \mathrm{C}$ freezer.

2.2. Small RNA Library Preparation. Total RNA was isolated using TRIzol reagent (Invitrogen). Approximately $5 \mu \mathrm{g}$ of total RNA from transgenic and control mice was submitted to the Beijing Genomics Institute (BGI) for Solexa sequencing. In brief, polyacrylamide gel electrophoresis (PAGE) technique was used to fractionate total RNA in the range of 16-30 nt, for sequencing. Fragments were then ligated with proprietary adapters. cDNA was synthesized from total RNA by reverse transcription and amplified to produce libraries for sequencing.

2.3. Analysis of the Output Data. The data as obtained in fastq format was converted to fastq2fast.pl; and revalidated clean miRNA-seq dataset was analyzed using mirDeep software v.2.1.2. The Mus musculus genome Mus_musculus. GRCm38.74 was downloaded from Ensembl, and the miRNA reference was obtained from the miRBase database (version 20).

The miRNA expression level of each library was normalized by the following formula:

$$
\begin{aligned}
& \text { Normalized reads count } \\
& \quad=\text { Reads count } \times 1,000,000 \text { total clean reads count. }
\end{aligned}
$$

The Student's $t$-test was used to calculate the $P$ value and analyze the expression difference between different groups [59]. The $q$ value was calculated by fdrtool in $R$ package [60].

2.4. Novel miRNA Prediction. The known and novel miRNAs were identified from deep sequencing data using miRDeep software v.2.1.2 package [61]. All sequences were mapped to the mouse genome using megaBLAST, and exactly matched sequences were processed for further analysis. Potential precursor miRNA sequences were extracted from the mouse genome and the secondary structures were predicted by RNAfold [62] (Figure 4).
2.5. Target Prediction. To observe the potential function of miRNAs with significantly differential expression in two groups (transgenic versus wild-type control), we used miRDB (Version 6.2) to predict putative target genes of miRNAs with homologous human miRNAs and target prediction score $\geq 60$ [63] (http://mirdb.org/miRDB/index.html). Validated targets module of miRWalk was also used to investigate hosts experimentally verified target genes of differentially expressing miRNAs in the study (http://www.umm.uni-heidelberg.de/apps/zmf/mirwalk/) [64].

2.6. Gene Ontology and Pathway Analysis. Gene ontology analysis was performed by analyzing KEGG pathways using "database for annotation, visualization, and integrated discovery" (DAVID) website $[65,66]$ with the following parameters: Count $=2$ and EASE $=1.0$. "Count" means the threshold of minimum gene counts belonging to an annotation term, and "EASE" is a modified Fisher Exact $P$ value.

\section{Results}

3.1. Overview of the Sequencing Data. Total reads from control CN148-11303077 and CN150-9357529 and from MSTN transgenic mice TN126-9734519, TN135-10207035, and TN329-21774919 were obtained. After trimming the adaptor and low quality reads, only clean reads of high quality were used for further analysis, that is, 10479962-CN148, 8375356-CN150, 10130133-TN126, 9052728-TN135, and 9677365TN329 accounting for $92.72 \%, 89.50 \%, 93.24 \%, 92.99 \%$, and $94.81 \%$, respectively, of the original reads. The sequencing data were simplified by grouping all identical sequence reads together; therefore 536489 (CN148), 224550 (CN150), 228238 (TN126), 177471 (TN135), and 190093 (TN329) unique sequences were used for subsequent analysis (Table 1 ). The most abundant size class in small RNA sequences distribution was $22 \mathrm{nt}$, followed by 21 and $23 \mathrm{nt}$ (Figure 1), and these estimates were in the accordance with the known 21-23 nt range for miRNAs [67]. To assess the efficiency of deep sequencing for miRNA detections, all sequence reads were annotated and classified by analyzing the sequence tags in relation to the data from miRBase (miRBase V20). The sequence tag annotation demonstrated that known Mus muculus miRNAs accounted for 59\% of all sequence reads in the MSTN and control libraries (Figure 2). These results indicate that the deep sequencing data were highly enriched 


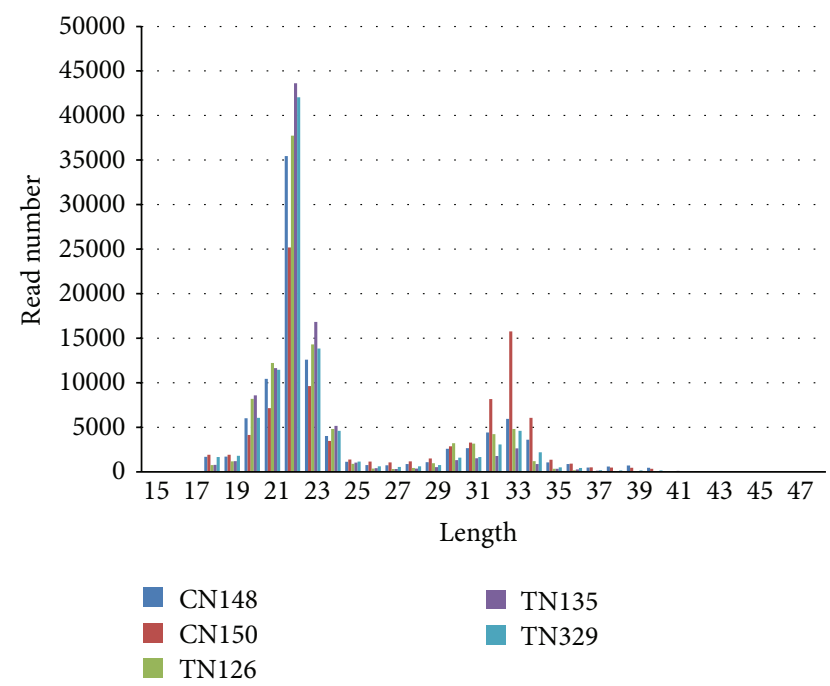

FIGURE 1: miRNA length distribution graph: miRNA length distribution graph depicting that most of all miRNA was consistent length of $22 \mathrm{nt}$.

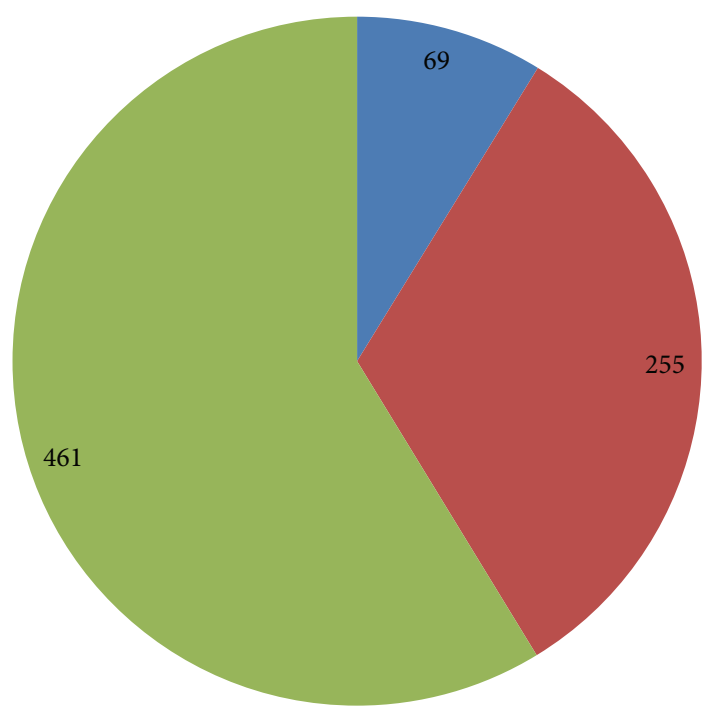

- Novel miRNAs which are not homologous with other species

- Novel miRNAs which are homologous with other species

- Known miRNAs

FIgURE 2: Annotation of miRNAs showing the number of known, homologous novel, and nonhomologous miRNAs.

for mature miRNA sequences, suggesting that the data are reliable for expression profiling of known miRNAs and deep mining for novel miRNAs.

Comparative expression of miRNAs in transgenic and control mice skeletal muscles revealed that 461 miRNAs obtained in the sequencing data matched perfectly with 1908 known Mus musculus miRNAs in the miRBase.
3.2. miRNA Transcriptome Analysis Demonstrated the Presences of Several Highly Abundant miRNAs in Mouse Skeletal Muscle. Almost all known muscle-specific miRNAs (myomiRs) of the more abundant miRNAs were identified in the analyzed muscle samples. The most abundant expressed miRNA was mmu-miR-22a which was represented by approximately 1637847 and 924589 reads in the small RNA libraries of the wild-type mice (CN148 and CN150) and 755381,1123582 , and 3994285 reads in the small RNA libraries of transgenic mice (TN126, TN135, and TN 329), respectively (Table 2, see Additional File 1 in the Supplementary Materials available online at http://dx.doi.org/10.1155/2014/328935). The predominance of miR-133a was consistent with its well established function during skeletal muscle development; miR-378a is also reported to play important role during Mus musculus myogenesis. Two other myomiRs, miR-26a, and miR-27b were high-count sequences in both libraries of transgenic and wild-type mice. MyomiR 208 and 499 were expressed at a very low level in current study.

3.3. Identification of miRNA Isoforms. The miRNAs also represent heterogeneity at $5^{\prime}$ and $/ 3^{\prime}$ end (Figure 3 ) and these variations from their miRBase reference sequences are referred to as isomiRs $[68,69]$. Examples of isomiRs are presented in Figure 3. In one case, the majority isoform, mmumiR-16a-2, the most abundant isoform is identical to the reference in miRBase (Figure 3, Additional File 2). In some other cases, such as mmu-miR-181b-2, mmu-miR-199a-2, and mmu-miR-16a-2, more than one highly abundant isoform was present (Figure 3), indicating that some miRNAs have more than one isoformin specific tissues.

3.4. Novel miRNAs Were Less Abundant and Less Conserved. In addition to profiling known miRNAs, deep sequencing is a powerful strategy for discovering novel miRNAs that may not have been detected by traditionalmethods from sequencing cDNA libraries. We identified a total of 69 putative novel Mus musculus miRNAs from wild-type controls and transgenic mouse sequence tags (Additional File 3). The putative novel miRNAs were less abundant than known miRNAs (Additional File 3). Two novel miRNAs NMmu-14 and NMmu36 having more than 100 read counts were validated and NMmul4 was found to be relatively abundant in comparison with miR NMmu36 (Figure 4) but both of these had read counts greater than 100 in the small RNA library from TN 329 library (Table 3). The novel miRNAs were less evolutionarily conserved (Additional File 3).

3.5. Identifications of Differentially Expressed miRNAs in Hypertrophic Muscle. To identify miRNAs associated with skeletal muscle hypertrophy, we compared the miRNA transcriptoms of skeletal muscle between MSTN propeptide transgenic mice and their littermate control mice. Of the 461 known miRNAs, 57 differentially expressed miRNAs were identified (Additional File 4). The miRNAs, which satisfied the criteria $\log 2$ FoldChange $\geq 1$ or $\leq-1$ and $P \leq 0.05$, $Q$ value 0.1 , were denoted as differentially expressed miRNAs. Cluster 
TABLE 2: Highly abundant known miRNAs in control and transgenic libraries.

\begin{tabular}{|c|c|c|c|c|c|c|}
\hline \multirow[b]{2}{*}{ miRNA } & \multicolumn{5}{|c|}{ Reads } & \multirow[b]{2}{*}{$\begin{array}{c}\text { Previous studies in } \\
\text { skeletal muscle }\end{array}$} \\
\hline & CN148 & CN150 & TN126 & TN135 & TN329 & \\
\hline mmu-miR-22-3p & 1637847 & 924589 & 1186390 & 755381 & 1123582 & \\
\hline mmu-miR-133a-3p & 1143874 & 716877 & 984332 & 1004715 & 1049011 & {$[38]$} \\
\hline mmu-miR-486-3p & 694107 & 288434 & 927009 & 902799 & 1325315 & [49] \\
\hline mmu-miR-143-3p & 496985 & 195585 & 820161 & 849193 & 676631 & \\
\hline mmu-miR-378a-3p & 427775 & 201482 & 420654 & 356711 & 417747 & {$[50]$} \\
\hline mmu-miR-10b-5p & 326382 & 192684 & 699540 & 645680 & 909894 & \\
\hline mmu-miR-26a-5p & 265020 & 151950 & 297146 & 184254 & 263216 & {$[51]$} \\
\hline mmu-miR-27b-3p & 190947 & 85527 & 204496 & 151307 & 174403 & {$[52,53]$} \\
\hline mmu-miR-10a-5p & 106751 & 35960 & 218660 & 179584 & 198961 & \\
\hline mmu-miR-126a-5p & 104630 & 34633 & 173507 & 98023 & 148161 & \\
\hline mmu-let-7f-5p & 76751 & 57966 & 85569 & 62527 & 66748 & \\
\hline mmu-miR-30d-5p & 71324 & 36990 & 71091 & 53116 & 69360 & \\
\hline mmu-miR-30e-5p & 46295 & 22850 & 77046 & 49869 & 64934 & \\
\hline mmu-miR-191-5p & 43014 & 20310 & 61773 & 53825 & 66552 & \\
\hline mmu-miR-30c-5p & 37487 & 18170 & 36383 & 25658 & 36686 & \\
\hline mmu-miR-16-5p & 28759 & 13306 & 33389 & 30684 & 29020 & \\
\hline mmu-miR-101a-3p & 27883 & 12254 & 42753 & 32687 & 43902 & {$[54]$} \\
\hline mmu-let-7i-5p & 27804 & 25012 & 21811 & 16454 & 15724 & \\
\hline mmu-miR-100-5p & 26629 & 8764 & 62824 & 58392 & 50326 & \\
\hline mmu-miR-1a-1-3p & 25824 & 4872 & 60020 & 59300 & 56369 & {$[55]$} \\
\hline mmu-miR-21a-5p & 25762 & 13328 & 32285 & 31527 & 22657 & {$[56]$} \\
\hline mmu-let-7a-5p & 22645 & 14527 & 26374 & 19001 & 18235 & \\
\hline mmu-miR-125a-5p & 22035 & 10736 & 20767 & 16909 & 13863 & {$[57]$} \\
\hline mmu-miR-127-3p & 20888 & 19550 & 42560 & 28964 & 36344 & \\
\hline mmu-miR-125b-5p & 18733 & 10574 & 17810 & 18525 & 12921 & [57] \\
\hline mmu-miR-26b-5p & 18498 & 7630 & 27992 & 20838 & 26573 & \\
\hline mmu-miR-378d-5p & 17270 & 7758 & 20914 & 14457 & 17438 & \\
\hline mmu-miR-133b-3p & 17250 & 7097 & 41049 & 66977 & 46252 & {$[38]$} \\
\hline mmu-miR-92a-1-3p & 16783 & 12552 & 11462 & 14209 & 10021 & \\
\hline mmu-miR-148a-3p & 16589 & 9216 & 20184 & 35176 & 17861 & [58] \\
\hline
\end{tabular}

TABLE 3: Abundantly expressed novel miRNAs in control and transgenic libraries.

\begin{tabular}{lccccccc}
\hline \multirow{2}{*}{ Name } & Mature sequence & & & Reads & & Chromosome position & Strand \\
& & CN148 & CN150 & TN126 & TN135 & TN329 & \\
\hline NMmu-14 & ugauuggaagacacucugcaaca & 0 & 0 & 159 & 129 & 0 & 16 \\
NMmu-36 & gauucggcugaucuggcuggc & 457 & 0 & 0 & 0 & 515 & 14 \\
\hline
\end{tabular}

analysis was generated to check the expression of miRNAs in different samples (Figure 5).

To validate the differentially expressed miRNAs of the wild-type control and transgenic mice, 8 known miRNAs and 2 novel miRNAs were randomly selected and their expression levels quantified by using real-time RT-PCR (Figures 4 and
$6)$. Of the 8 known miRNAs examined, 7 miRNAs (miR425, miR-26a, miR-1a, miR-199a, miR-101, miR-378, and miR151) showed a consistent pattern with the deep sequencing data (Figures 6(a)-6(j)). These data demonstrated that deep sequencing is a sensitive and reliable method for identifying differentially expressed miRNAs. 
mmu-miR-16a-2

$5^{\prime}$-caugcuuguuccacucuagcagcacguaaauauuggcguagugaaauaaauauuaaacac- $3^{\prime} \exp$ $\ldots(((((.((((\ldots)((.((.((\ldots \ldots))))))))))))))).) \ldots \ldots \ldots$ reads $\mathrm{mm}$

$\ldots \ldots \ldots \ldots$. . . .

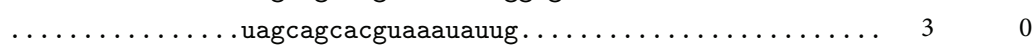

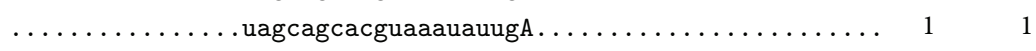

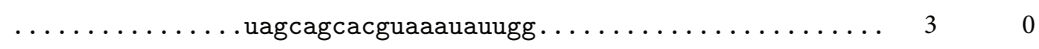

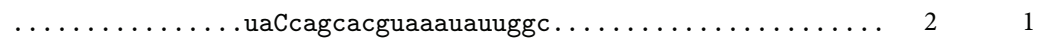

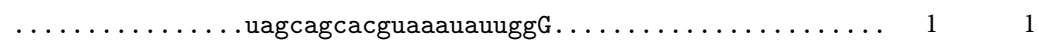

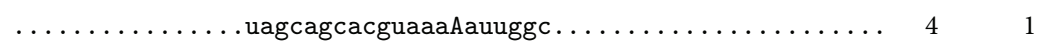

$\ldots \ldots \ldots \ldots$. . . . . .

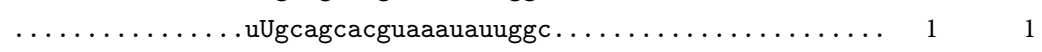

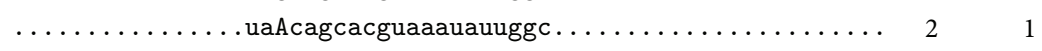

$\ldots \ldots \ldots \ldots \ldots$ uagcagcGcguaaauauuggc $\ldots \ldots \ldots \ldots \ldots \ldots \ldots, 1$

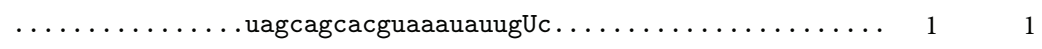

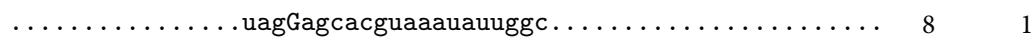

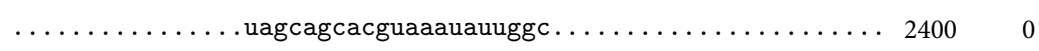

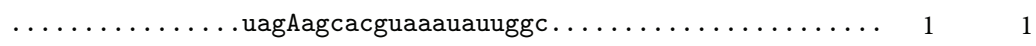

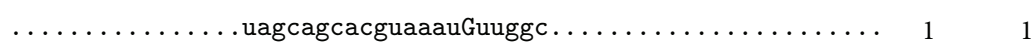

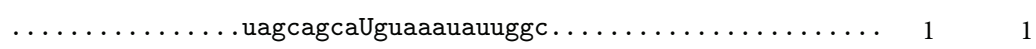

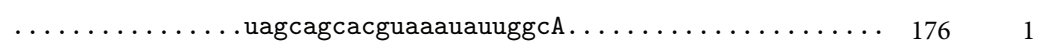

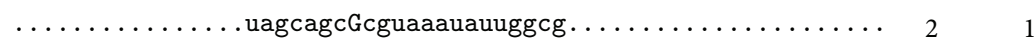

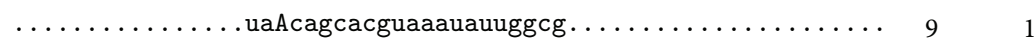

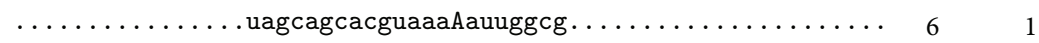

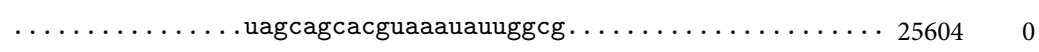

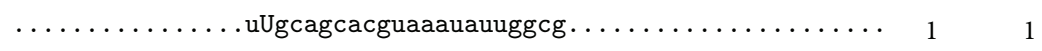

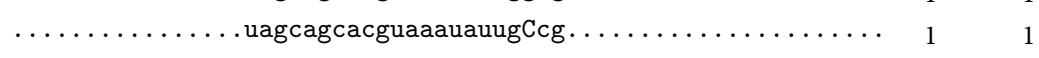

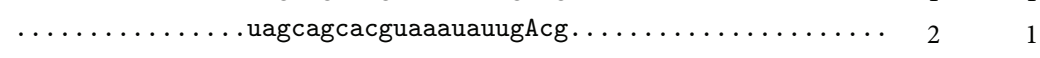

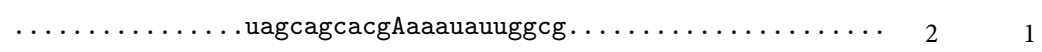

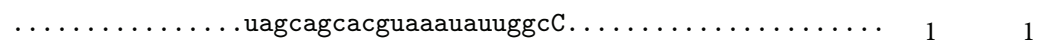

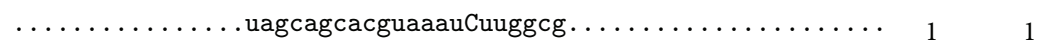

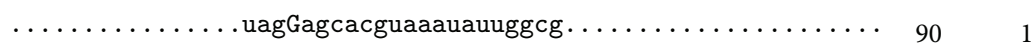

$\ldots \ldots \ldots \ldots \ldots$ uagcagcacguaaauauuggcgA................. $87 \quad 1$

mmu-miR-133a-1

$5^{\prime}$-gcuaaagcugguaaaauggaaccaaaucgccucuucaauggauuugguccccuucaacca- $3^{\prime} \exp$ $((\ldots .)) ..(((\ldots((.((.(((((((\ldots \ldots \ldots \ldots))))))))))).) \ldots)))$. reads

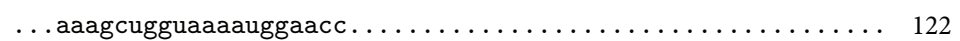

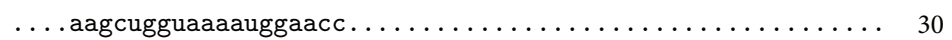

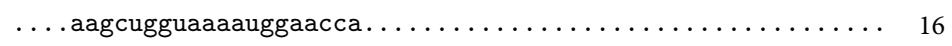

$\ldots \ldots$ aagcugguaaaauggaaccaa . . . . . . . . . . . . . . . 104

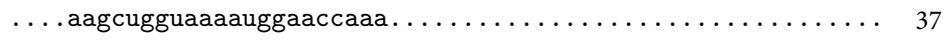

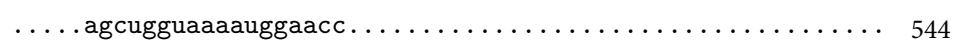

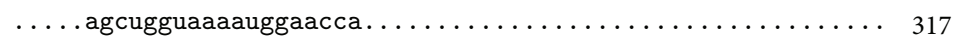

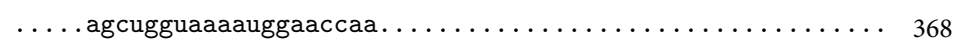

... agcugguaaaauggaaccaaa. . . . . . . . . . . . . . . . . 2409

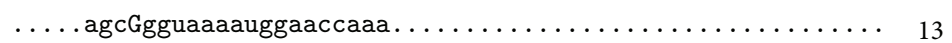

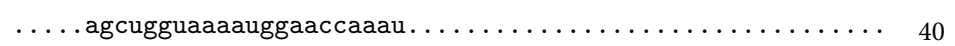

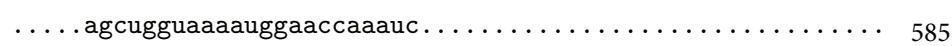

$\ldots \ldots$ agcugguaaaauggaaccaaaucgcc . . . . . . . . . . . . 80

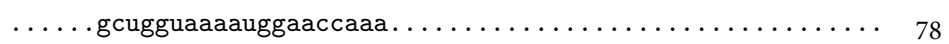

$\ldots \ldots$ gcugguaaaauggaaccaaauc . . . . . . . . . . . . . . . 106

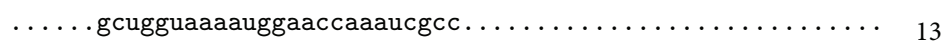

(a)

Figure 3: Continued. 
mmu-miR-188b-2

$5^{\prime}$-uugauggcugcacucaacauucauugcugucgguggguuugaaugucaaccaacucacug- $3^{\prime} \exp$ $.(((((.((((.((((.((((((((\ldots))))))))))))))))))).) \ldots))) \ldots$ reads $\mathrm{mm}$

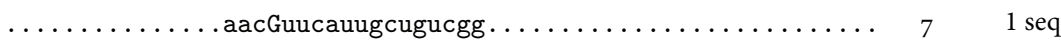

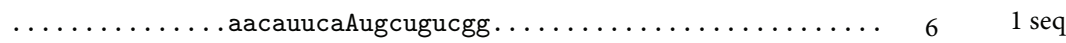

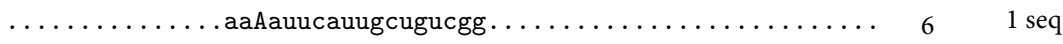

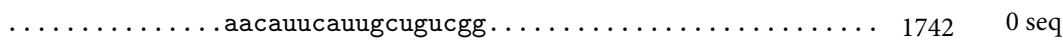

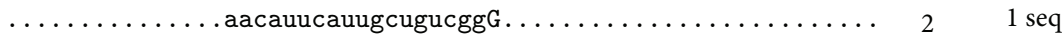

$\ldots \ldots \ldots \ldots$ aacauucauugcugucggu.................. $50 \quad 0$ seq

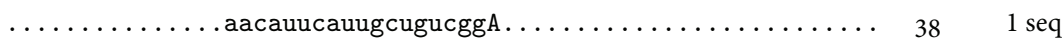

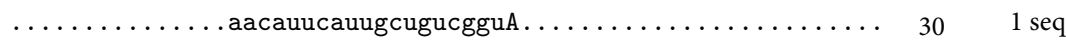

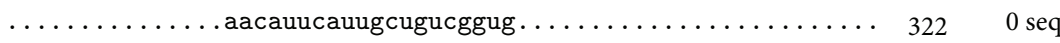

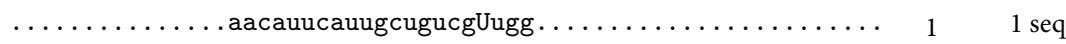

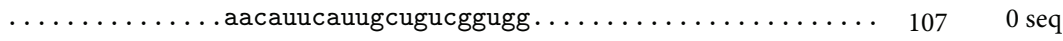

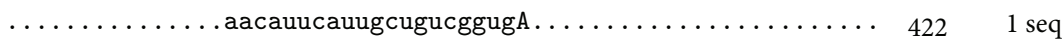

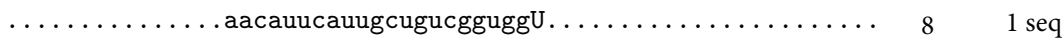

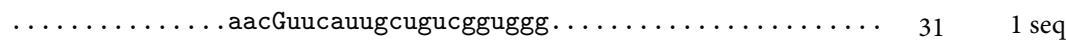

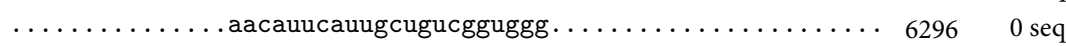

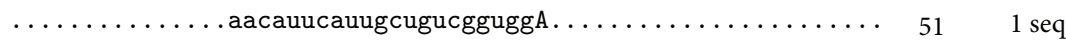

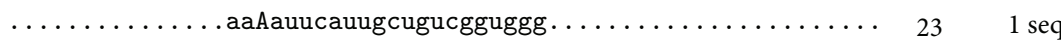

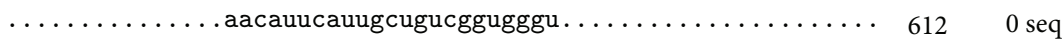

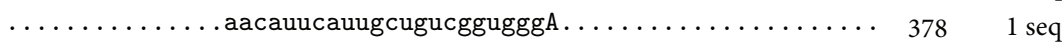

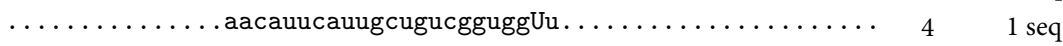

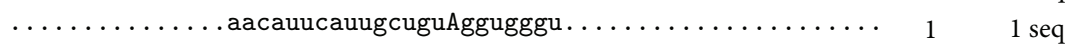

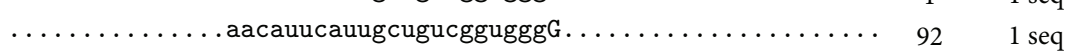

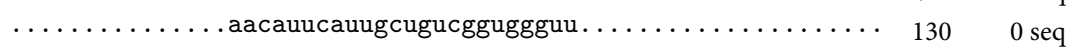

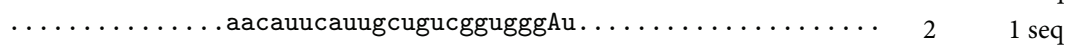

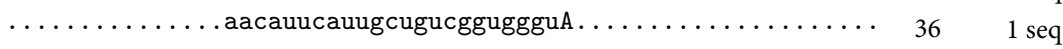

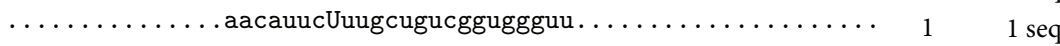

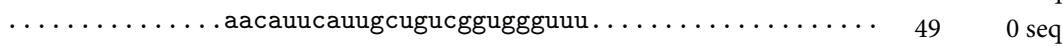

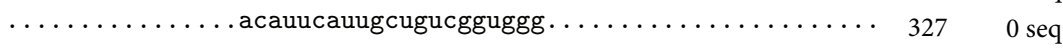

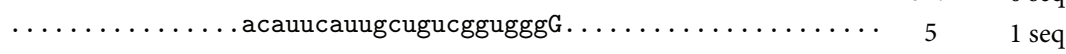

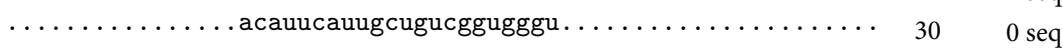

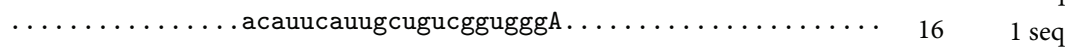

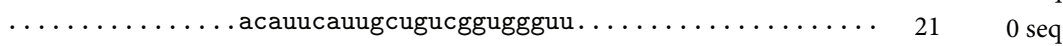

$5^{\prime}$-mmu-miR-199a-2

uggaagcuucaggagauccugcuccgucgccccaguguucagacuaccuguucaggacaa-3' exp $(((\ldots((\ldots . .(((\ldots \ldots)))) \ldots)))).) .(((((.(((\ldots \ldots))) \ldots)))))$. reads $\mathrm{mm}$

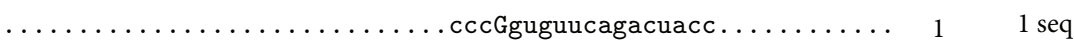

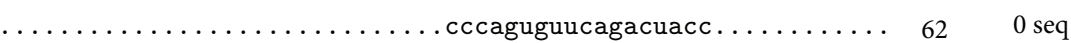

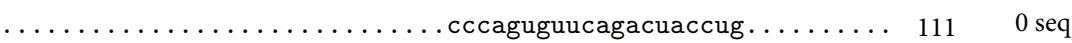

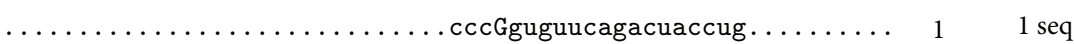

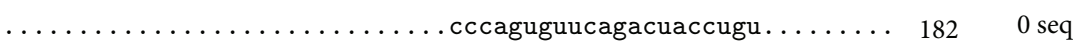

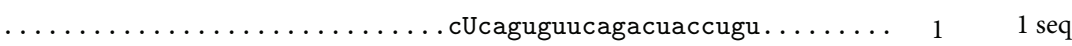

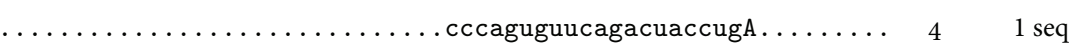

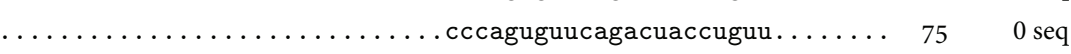

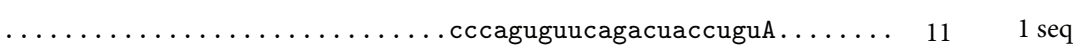

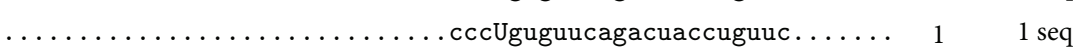

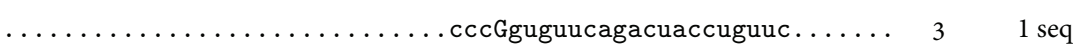

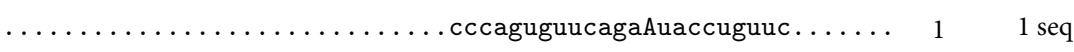

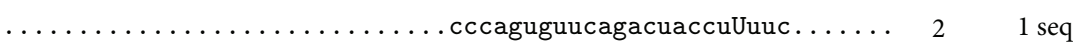

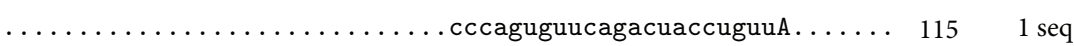

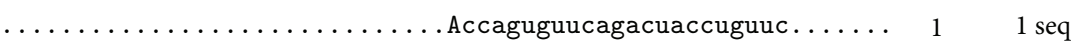

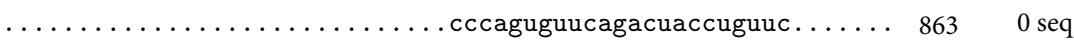

(b)

Figure 3: Continued. 


\begin{tabular}{|c|c|c|}
\hline$\ldots \ldots$. cса & 1 & $1 \mathrm{se}$ \\
\hline$\ldots \ldots \ldots \ldots \ldots \ldots \ldots$ cAcaguguucagacuaccuguuc . . . . & 1 & $1 \mathrm{seq}$ \\
\hline 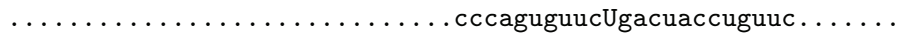 & 1 & \\
\hline$\ldots \ldots \ldots \ldots \ldots \ldots \ldots \ldots$. . . . . . . . . . & 3 & \\
\hline$\ldots \ldots \ldots \ldots \ldots \ldots \ldots \ldots \ldots \ldots \ldots \ldots \ldots \ldots$ ccaguguucGgacuaccuguuc. . . & 1 & \\
\hline 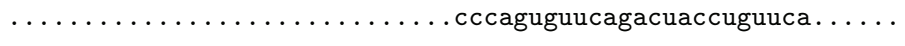 & 30 & \\
\hline 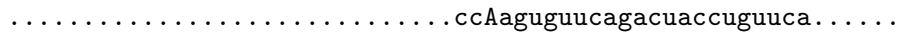 & 1 & \\
\hline 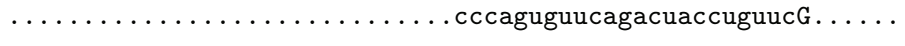 & 1 & \\
\hline$\ldots \ldots \ldots \ldots \ldots \ldots \ldots \ldots \ldots \ldots \ldots \ldots \ldots \ldots$ ccaguguucagacuaccuguucaA.... & 1 & \\
\hline -mmu-miR-486 & & \\
\hline agccagcucugaucucgcccucccugagggguccu & $\mathrm{xp}$ & \\
\hline$\ldots((((((\ldots((\ldots(((((\ldots \ldots)))).) \ldots)) \ldots .))))).) \ldots \ldots \ldots \ldots$ & reads & \\
\hline$\ldots \ldots \ldots \ldots \ldots$ gUgguccuguacugagcugccсc........ & 1 & \\
\hline 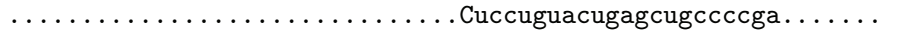 & 139 & \\
\hline 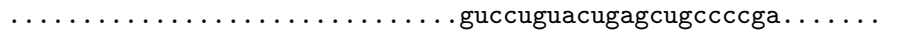 & 13 & \\
\hline$\ldots \ldots \ldots \ldots \ldots \ldots \ldots$ Cuccuguacugagcugccccgag . . . & 614 & \\
\hline 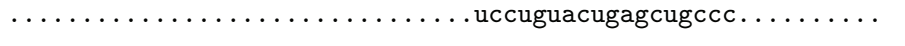 & 236 & \\
\hline 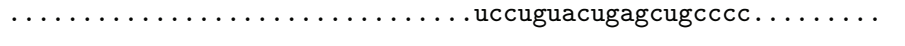 & 714 & \\
\hline 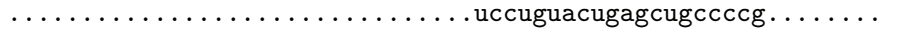 & 7622 & \\
\hline 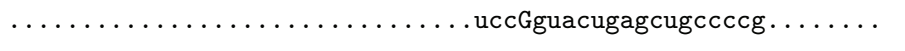 & 108 & \\
\hline$\ldots \ldots \ldots \ldots$ ucAuguacugagcugccccg $\ldots \ldots \ldots$ & 42 & \\
\hline$\ldots \ldots \ldots \ldots$............. & 1139 & \\
\hline$\ldots \ldots \ldots$...... uccGguacugagcugccccga...... & 723 & \\
\hline$\ldots \ldots \ldots$ ucAuguacugagcugccccga....... & 390 & \\
\hline ...uccuguacugagcugccccga..... & 98430 & \\
\hline ...uccuguacugagcugccccgaC..... & 974 & \\
\hline ........uccuguacugagcugccccgGg. . & 624 & \\
\hline .......uccuguacugagcugcccUgag. & 83 & \\
\hline$\ldots \ldots \ldots \ldots \ldots$ uccuguacugagcugccccgCg . . . . & 25 & \\
\hline$\ldots \ldots \ldots \ldots \ldots \ldots \ldots$ uccuguacugagcugAcccgag . . . . & 129 & \\
\hline$\ldots \ldots \ldots$ uccuguacugagcugcAccgag. ..... & 60 & \\
\hline 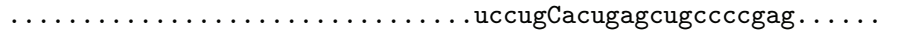 & 79 & \\
\hline$\ldots \ldots \ldots \ldots \ldots \ldots \ldots \ldots \ldots \ldots \ldots$ ucAuguacugagcugccccgag $\ldots \ldots$ & 2018 & \\
\hline$\ldots \ldots \ldots \ldots \ldots \ldots \ldots \ldots$ ucGuguacugagcugccccgag . . . . & 186 & \\
\hline 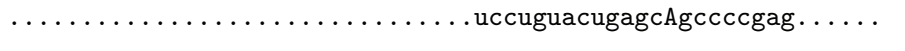 & 124 & \\
\hline$\ldots \ldots \ldots \ldots \ldots \ldots$ uccuguacugaCcugccccgag..... & 30 & \\
\hline$\ldots \ldots \ldots \ldots \ldots \ldots \ldots$. $\ldots \ldots$ ucuguacugagcugccccgag . . . . & 116 & \\
\hline$\ldots \ldots \ldots \ldots \ldots \ldots \ldots \ldots \ldots \ldots$ uccGguacugagcugccccgag . . . . & 3561 & \\
\hline$\ldots \ldots \ldots \ldots \ldots$ uccuguacugagcugccccgaU. ..... & 596 & \\
\hline$\ldots \ldots \ldots$. nccuguacug & 55 & \\
\hline ...uccuguacugagcCgccccgag...... & 79 & \\
\hline gcugccccgaA ..... & 4421 & \\
\hline Cccccgag...... & 150 & \\
\hline ....uccuguacugagcugccc & 507280 & \\
\hline ...uccuguacugagcugccccgagA ..... & 53390 & \\
\hline ....uccuguacugagcugccccgagg..... & 2228 & \\
\hline$\ldots \ldots \ldots \ldots \ldots$ uccuguacugagcugccccgagu. ... & 8278 & \\
\hline ........uccuguacugagcugccccgaggu.... & 191 & \\
\hline$\ldots$...uccugu & 556 & \\
\hline$\ldots \ldots \ldots \ldots$ uccug & 73 & \\
\hline
\end{tabular}

(c)

Figure 3: Continued. 


\begin{tabular}{|c|c|c|}
\hline$\ldots \ldots \ldots \ldots \ldots \ldots \ldots$ uccuguacugagcugccccgagUu.... & 4893 & $1 \mathrm{seq}$ \\
\hline$\ldots \ldots \ldots \ldots \ldots$. . . . . . . & 129 & $1 \mathrm{seq}$ \\
\hline$\ldots \ldots \ldots \ldots \ldots \ldots$ uccuguacugagcugccccgagguU. . & 128 & $1 \mathrm{seq}$ \\
\hline$\ldots \ldots \ldots \ldots \ldots \ldots \ldots \ldots$ cuguacugagcugccccgag . . . . & 374 & 0 seq \\
\hline$\ldots \ldots \ldots \ldots \ldots \ldots \ldots \ldots$ cuguacugagcugccccgagg. . . . & 1040 & 0 seq \\
\hline$\ldots \ldots \ldots \ldots \ldots \ldots$ cuguacugagcugccccgaggu. ... & 194 & 0 seq \\
\hline$\ldots \ldots \ldots \ldots \ldots \ldots$ cuguacugagcugccccgagguU. . & 163 & $1 \mathrm{seq}$ \\
\hline 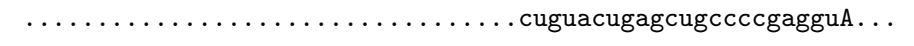 & 274 & $1 \mathrm{seq}$ \\
\hline mmu-miR-26b & & \\
\hline $5^{\prime}$-ugcccgggacccaguucaaguaauucaggauagguuguggugcugaccagccuguucucc & & $\mathrm{mm}$ \\
\hline$\ldots \ldots \ldots \ldots$ uucaagAaauucaggauaggu . . . . . . . . . . . . . & 8 & $1 \mathrm{seq}$ \\
\hline$\ldots \ldots \ldots \ldots$. . . . . . . . . . . . . . . . . & 2 & $1 \mathrm{seq}$ \\
\hline$\ldots \ldots \ldots \ldots$. . . uucaaguaauuUaggauaggu . . . . . . . . . . . . . . & 8 & $1 \mathrm{seq}$ \\
\hline ...........uucaaUuaauucaggauaggu. . . . . . . & 3 & $1 \mathrm{seq}$ \\
\hline .......... . uuUaaguaauucaggauaggu. . . . . . & 2 & $1 \mathrm{seq}$ \\
\hline ..........uucaaguaauucaAgauaggu. ....... & 5 & $1 \mathrm{seq}$ \\
\hline$\ldots \ldots \ldots$......uucaaguaauucaggauaggC . . . . . . . & 269 & $1 \mathrm{seq}$ \\
\hline$\ldots \ldots \ldots \ldots$. . uucaaguaGuucaggauaggu. . . . . . . . . . . . . & 2 & $1 \mathrm{seq}$ \\
\hline ........... uucaaguaauucaggaCaggu. . . . . . . . . . . . . . . . & 2 & $1 \mathrm{seq}$ \\
\hline$\ldots \ldots \ldots \ldots$. . . uucaagCaauucaggauaggu. . . . . . . . . . . . . . & 5 & $1 \mathrm{seq}$ \\
\hline$\ldots \ldots \ldots \ldots$. . . Gucaaguaauucaggauaggu. . . . . . . . . . . . . . . . & 2 & $1 \mathrm{seq}$ \\
\hline$\ldots \ldots \ldots \ldots$. . . uucaaguaauucaggauaggu . . . . . . . . . . . . . . & 11195 & 0 seq \\
\hline$\ldots \ldots \ldots \ldots$. Aucaaguaauucaggauaggu . . . . . . . . . . . . . . & 3 & $1 \mathrm{seq}$ \\
\hline$\ldots \ldots \ldots \ldots$. . . uucaGguaauucaggauaggu . . . . . . . . . . . . . . . & 3 & $1 \mathrm{seq}$ \\
\hline$\ldots \ldots \ldots \ldots$. . uucaaguaauucaggauaggG. . . . . & 13 & $1 \mathrm{seq}$ \\
\hline$\ldots \ldots \ldots \ldots$. . uucaaguaauuAaggauaggu. . . . . . & 2 & $1 \mathrm{seq}$ \\
\hline$\ldots \ldots \ldots \ldots$. . uucaaAuaauucaggauaggu . . . . . . . . . . . . & 2 & $1 \mathrm{seq}$ \\
\hline$\ldots \ldots \ldots \ldots$ uuGaaguaauucaggauaggu . . . . . . . . . . . . . & 6 & $1 \mathrm{seq}$ \\
\hline$\ldots \ldots \ldots \ldots$. . uucaagGaauucaggauaggu . . . . . . . . . . . . & 4 & $1 \mathrm{seq}$ \\
\hline$\ldots \ldots \ldots \ldots$ uuAaaguaauucaggauaggu . . . . . . . . . . . . . & 42 & $1 \mathrm{seq}$ \\
\hline$\ldots \ldots \ldots \ldots$. . uucaaguaauCcaggauaggu . . . . . . . . . . . . . & 57 & $1 \mathrm{seq}$ \\
\hline$\ldots \ldots \ldots \ldots$. . . uucaaguaaCucaggauaggu. . . . . . . . . . . . . . & 1 & $1 \mathrm{seq}$ \\
\hline$\ldots \ldots \ldots \ldots$. . . uucaaguaauucaggauaAgu. . . . . . . . . . . . . . & 2 & $1 \mathrm{seq}$ \\
\hline$\ldots \ldots \ldots \ldots$. . . uucGaguaauucaggauaggu . . . . . . . . . . . . . & 46 & $1 \mathrm{seq}$ \\
\hline$\ldots \ldots \ldots \ldots$. . . uuAaaguaauucaggauagguu . . . . . . . . . . . . & 7 & $1 \mathrm{seq}$ \\
\hline$\ldots \ldots \ldots \ldots$. . uucaaguaauCcaggauagguu . . . . . . . . . . . . & 8 & $1 \mathrm{seq}$ \\
\hline$\ldots \ldots \ldots \ldots$. . . uucaaguaauucaggauaggCu. . . . . & 12 & $1 \mathrm{seq}$ \\
\hline ...........uucaaguaauucaggauagguA . . . . . & 528 & $1 \mathrm{seq}$ \\
\hline ..........uucaaguaauucaggauagguC... . & 3 & $1 \mathrm{seq}$ \\
\hline$\ldots \ldots \ldots$. . . uucaaguaauucaggauagguu... & 917 & $0 \mathrm{seq}$ \\
\hline ..........uGcaaguaauucaggauagguu. . . & 1 & $1 \mathrm{seq}$ \\
\hline ......uucaaguaauucaggauagguug. & 23 & 0 seq \\
\hline ..........uucaaguaauucaggauagguuC. . . & 29 & $1 \mathrm{seq}$ \\
\hline ...........uucaaguaauucaggauagguuU. . . . & 29 & $1 \mathrm{seq}$ \\
\hline$\ldots \ldots \ldots \ldots$ uucaaguaauucaggauagguAg $\ldots \ldots \ldots \ldots \ldots$ & 8 & $1 \mathrm{seq}$ \\
\hline$\ldots \ldots \ldots \ldots$. . . uucaaguaauucaggauagguuA . . . . . . . & 929 & $1 \mathrm{seq}$ \\
\hline$\ldots \ldots \ldots \ldots$ uucaaguaauucaggauagguugA $\ldots \ldots \ldots \ldots \ldots$ & 3 & $1 \mathrm{seq}$ \\
\hline$\ldots \ldots \ldots \ldots \ldots$ ucaaguaauucaggauaggu . . . . . . . . . . . . . & 3 & $0 \mathrm{seq}$ \\
\hline ...........ucaag & 2 & $1 \mathrm{seq}$ \\
\hline
\end{tabular}

(d)

FIGURE 3: IsomiRs from different miRNAs: reads alignments of the various isoforms of several mmu-miRs are presented. The sequence of the $\mathrm{mmu}-\mathrm{miR}$ hairpin is presented in the top line; the brackets below denote the secondary structure. Reads aligned with the mature mmu-miR sequence as reported in miRBase are denoted by a series of asterisks. The number of reads corresponding to each sequence is presented on the right. 


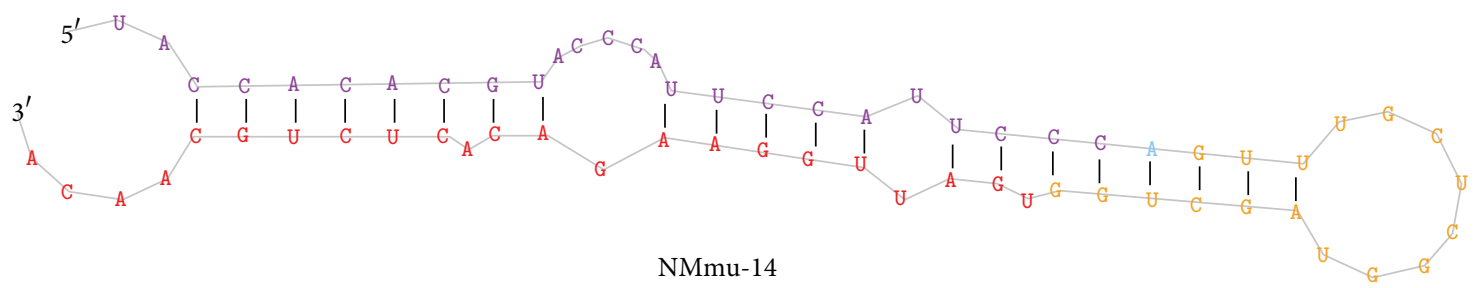

(a)

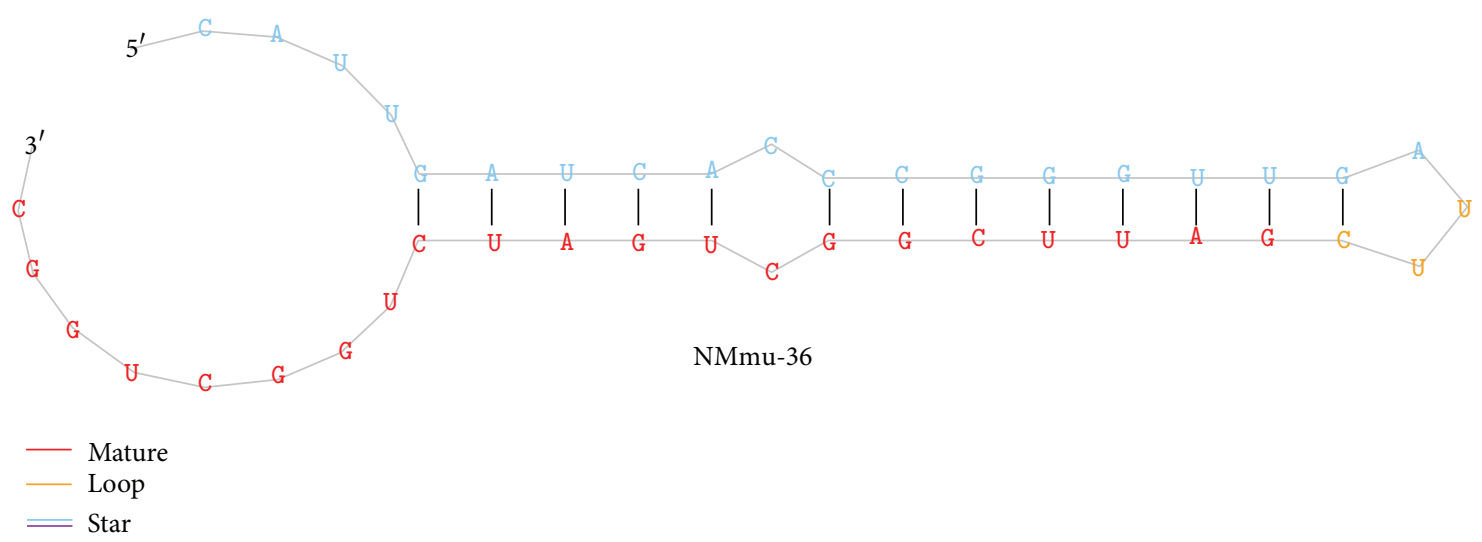

(b)

FIGURE 4: Predicted secondary structure of novel miRNAs: novel miRNAs secondary structure was predicted using miRDeep Software, where red color indicates the mature sequence, yellow color indicates loop sequence, blue color indicates the predicted star sequence, and purple color indicates miRNA star sequence.

3.6. Target Prediction for Differentially Expressed miRNAs. To ascertain the query how miRNAs function in concert with their target genes in muscle tissue, we identified targets of the differentially expressed miRNAs. Of the 57 differentially expressed miRNAs, 20 abundant miRNAs were selected to predict the targets and 4,413 annotated mRNA transcripts were identified (Additional File 5).

3.7. Gene Ontology and Pathway Analysis. Predicted 4,413 targets were analyzed for pathway analysis and the results showed that approximately 110 pathways are involved in corresponding miRNAs. Out of 110 pathways, 16 pathways are found to be associated with the most number of targeted genes (Figure 7), including mTOR signaling, insulinlike growth factor, MAPK signaling, and TGF- $\beta$ signaling. We also found that 20 differentially expressed miRNAs target many important genes such as FST, SMAD3, SMAD4, TGFBR1, ACRVR1a, ACVR1C, ACVR2a, ACVR, AKT3, and MEF2C (Table 4), which have been known to play a vital role in MSTN signalling pathway.

3.8. Results of Q-PCR Validation. Out of 57 differentially expressed miRNAs, 8 known miRNAs and 2 novel miRNAs were confirmed by using real-time RT-PCR (Figures 6(a)$6(j))$. Out of 8 known miRNAs, 6 miRNAs have been functionally linked to myogenesis (i.e., miR-1a, miR-26a, miR-133a and miR-199a, miR-101, and miR-378 [38, 50, 51, 54, 55, 70]). The levels of these miRNA expressions from Q-PCR analysis are consistent with the RNA sequence data in both wild-type and transgenic mice.

\section{Discussion}

4.1. miRNA Identifications from Myostatin Propeptide Transgenic and Wild-Type Mice. Direct sequencing of RNA molecules with next-generation sequencing (NGS) technology has revolutionized the analysis of transcriptome due to highthroughput scale and low cost. Recent development with Illumina's microRNA-Seq by Genome Analyzer System had enabled direct identification and profiling of microRNAs in various organisms [71]. The present study identified a total of 461 miRNAs, of which 57 were known and differentially expressed and 69 were novel in skeletal muscle tissue. Of the 57 differentially expressed miRNAs mmu-mir-133a was the most abundant in the wild-type control $(32,12,465.607)$ and transgenic $(57,10,658.17)$ mice. It is previously reported that miR-133a enhances myoblast proliferation by repressing serum response factor (SRF) [23]. miR-206 was demonstrated to be the most abundant miRNA in skeletal muscles of broilers (131,609 reads) and layers (222,998 reads) [48]. Considering these previous studies, we presume that miR-133a and miR-206 play an important role in skeletal muscle development. In our study, we observed that miR-206 expression level was significantly lower than miR-133a in comparison to previous studies. miR-206 is reported to be one of the skeletal muscle-specific myomiR and many studies have documented its pivotal role in skeletal muscle differentiation 

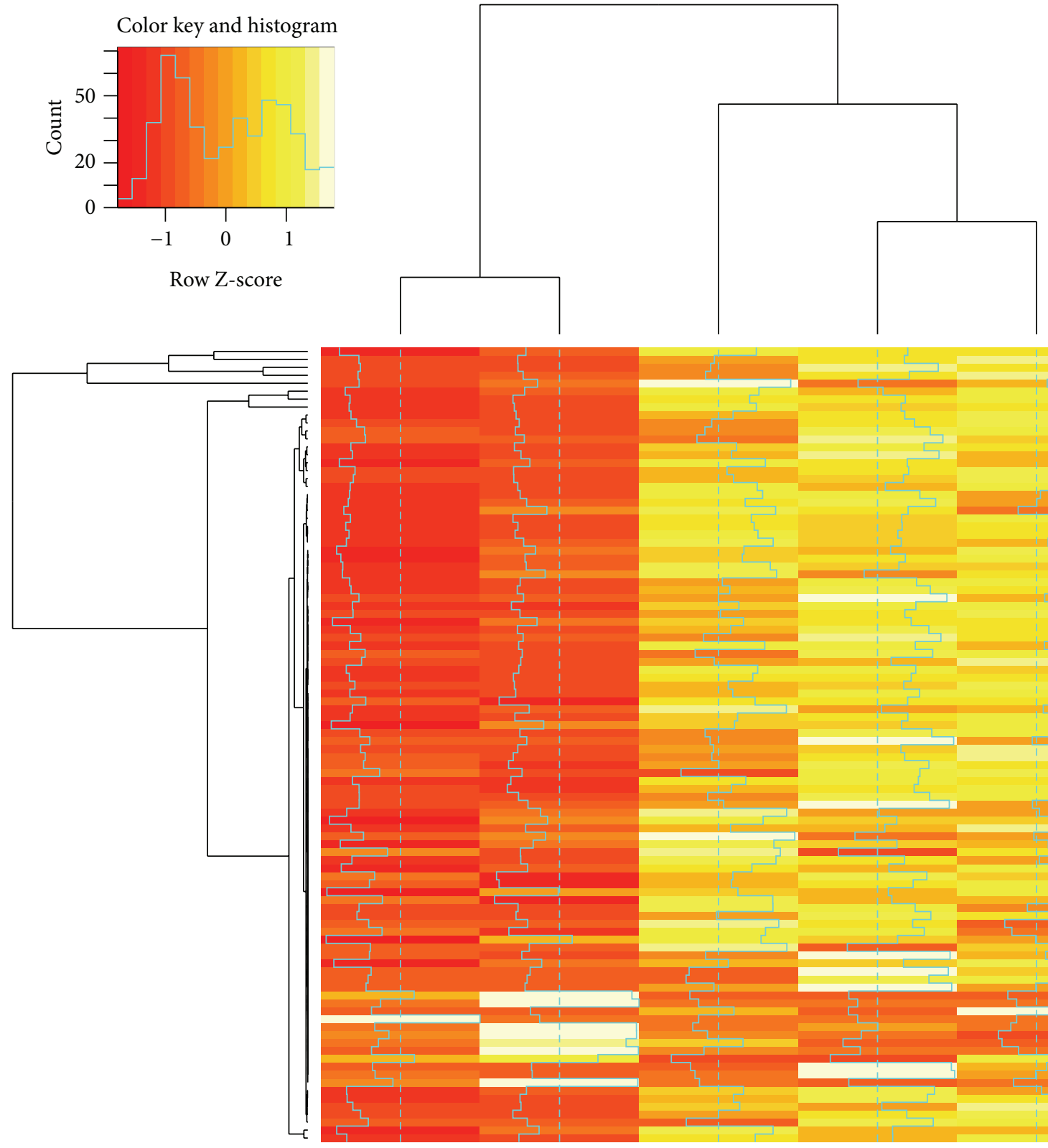

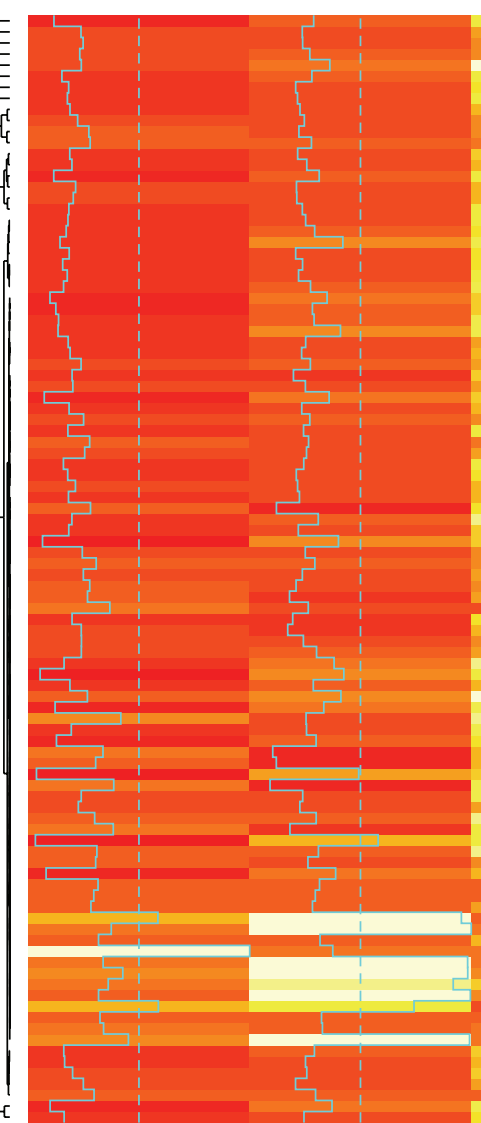

CN148

$\mathrm{CN} 150$
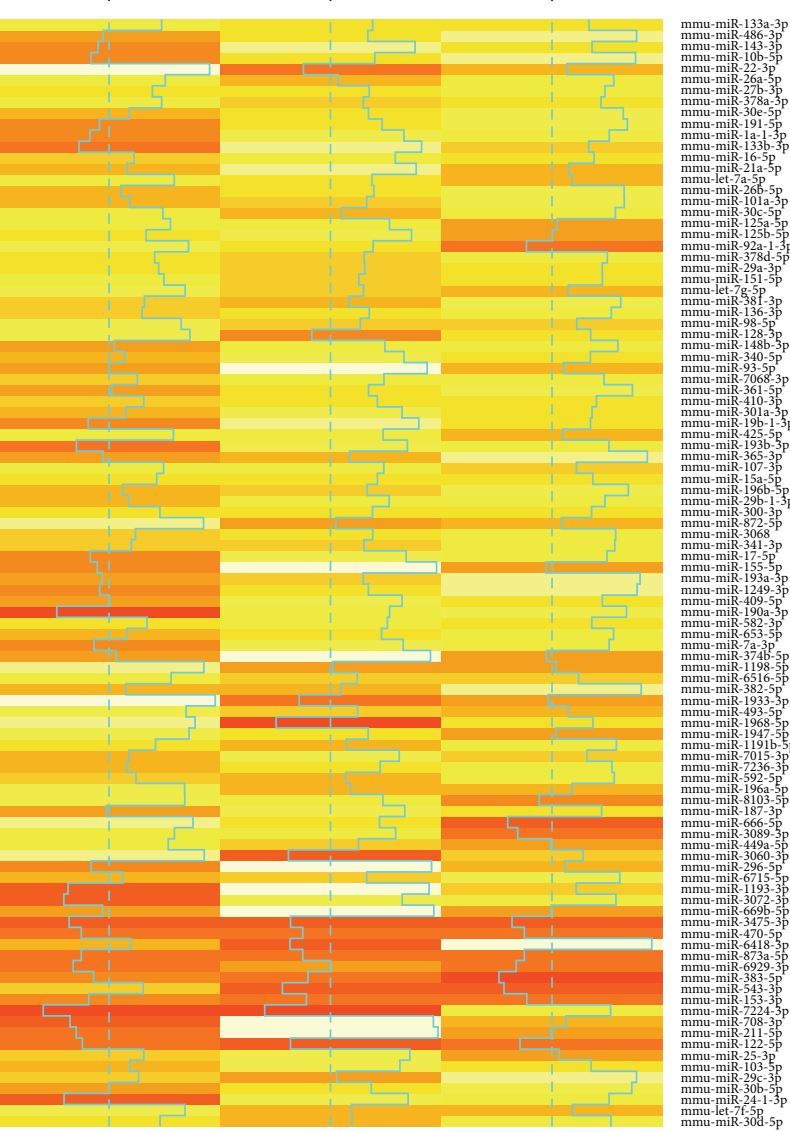

TN329

TN126

TN135

FIGURE 5: Identification of differentially expressed miRNAs in control and transgenic mice: Cluster analysis of differentially expressed miRNAs in control and transgenic mice based on the read counts obtained from deep sequencing data. CN: Control, TN: Transgenic.

[43, 49, 72-75] and miR-133a in regulating myogenesis by increasing muscle cell proliferation $[23,38,76]$. Differences in the expression levels of both the miRNAs (miR-133a and miR-206) in skeletal muscle libraries could reflect different roles of these miRNAs in terms of myogenesis regulation [77]. Similar to miR-133a and miR206, miR-1 also regulate muscle differentiation and development [23, 77-79]. miR1 and miR-133 modulate skeletal-muscle-cell proliferation and differentiation by repressing the activity of HDAC4 (histone deacetylase 4; a signal-dependent inhibitor of muscle differentiation) and SRF, respectively, thereby establishing negative-feedback loops for muscle-cell differentiation [23].

In addition to well-known myomiRs, recent studies have demonstrated that miR-486 [49], miR-378 [50], miR181a [80], miR-21a, miR-101a, and miR-151 [54] are also involved in regulation of myogenesis and several other ubiquitously expressed miRNAs have also been found to participate in myogenesis, including miR-26a [51], miR-27b [52, 53], and miR-29 [44]. All these nine miRNAs were also found to be abundantly expressed in our sequence libraries, which 


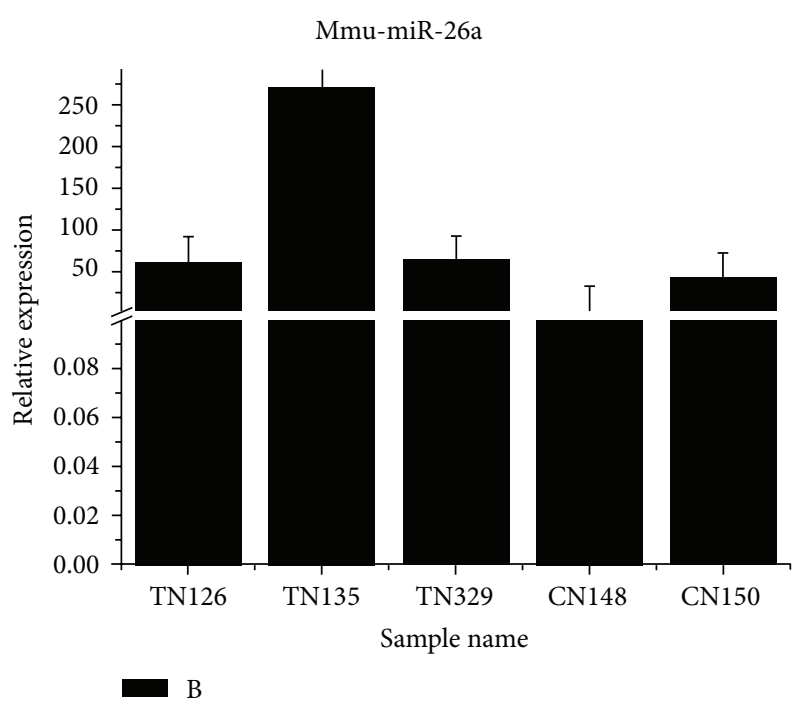

(a)

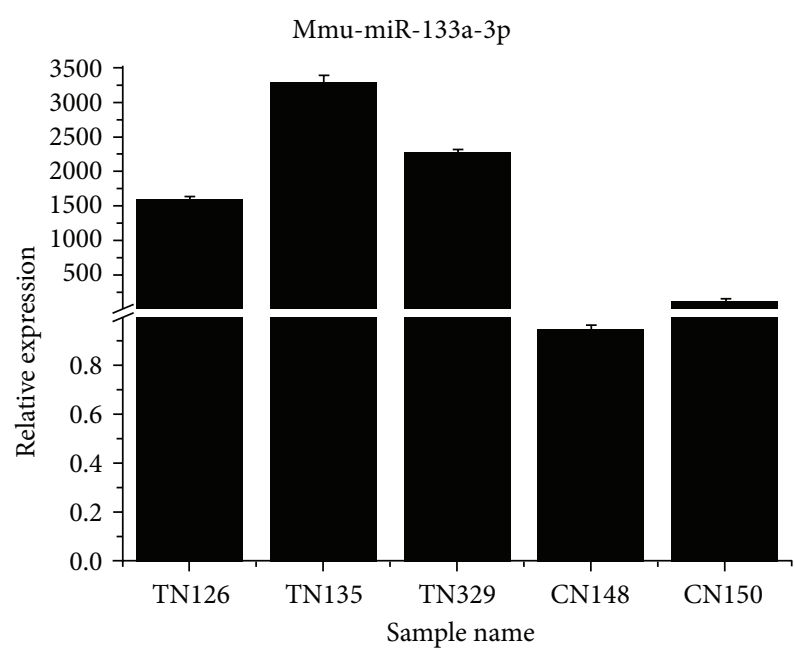

(c)

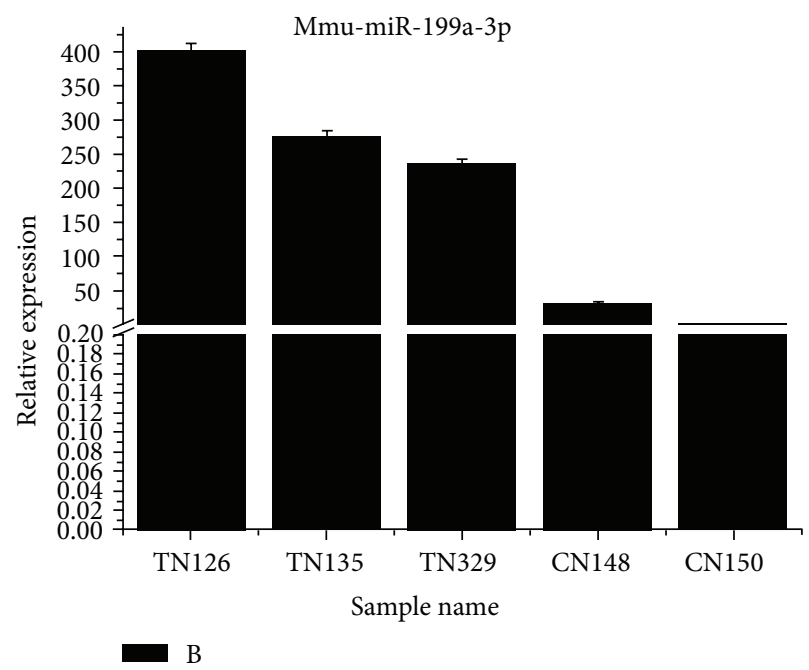

(e)

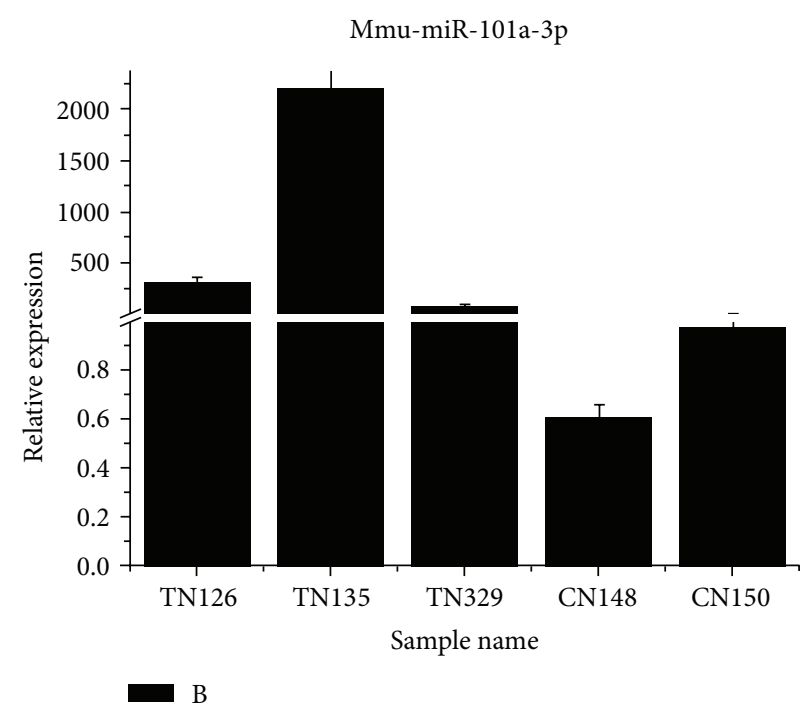

(b)

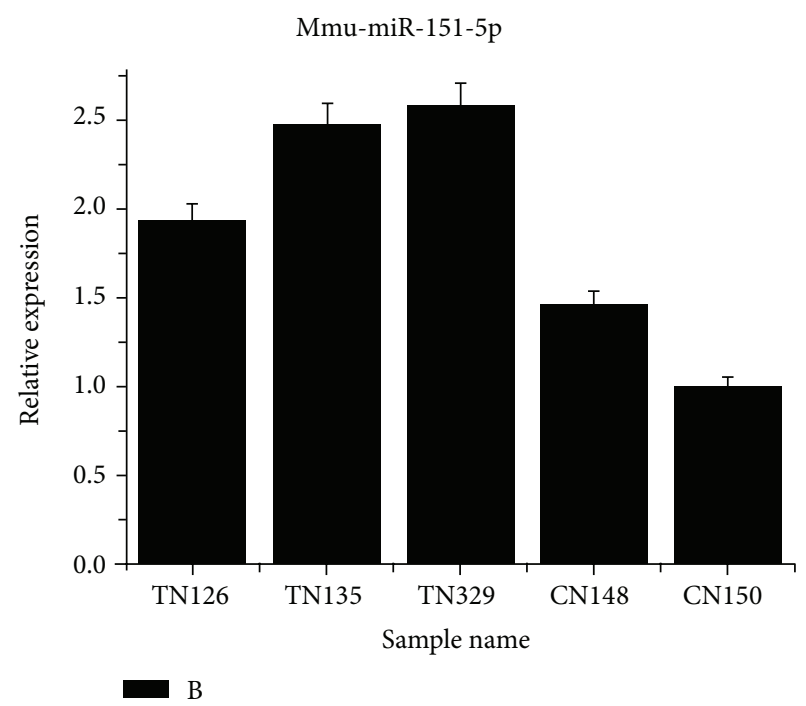

(d)

Mmu-miR-378a-3p

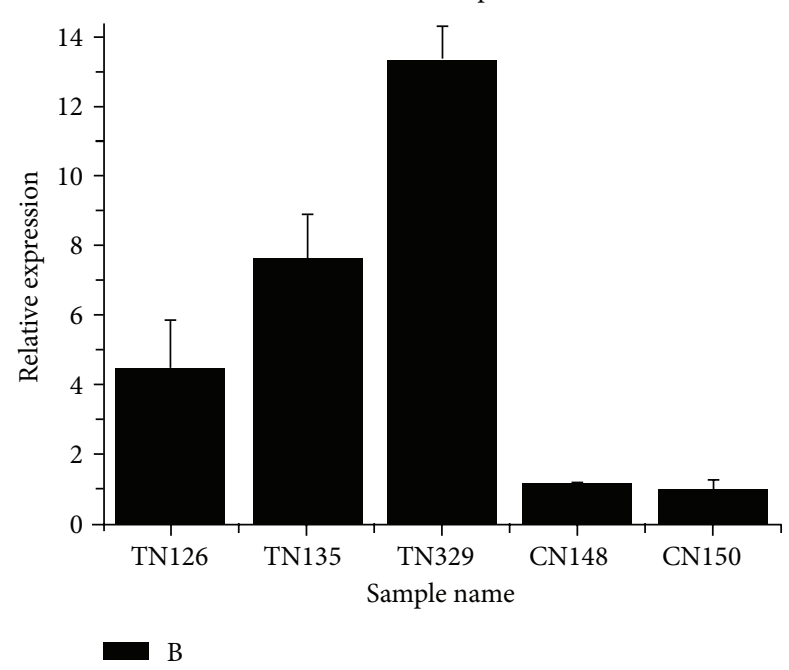

(f)

Figure 6: Continued. 


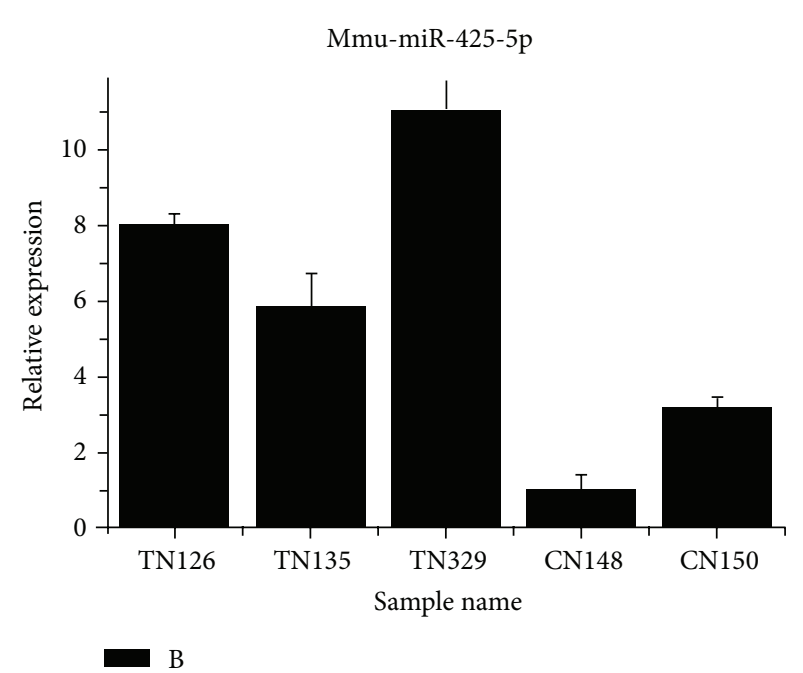

(g)

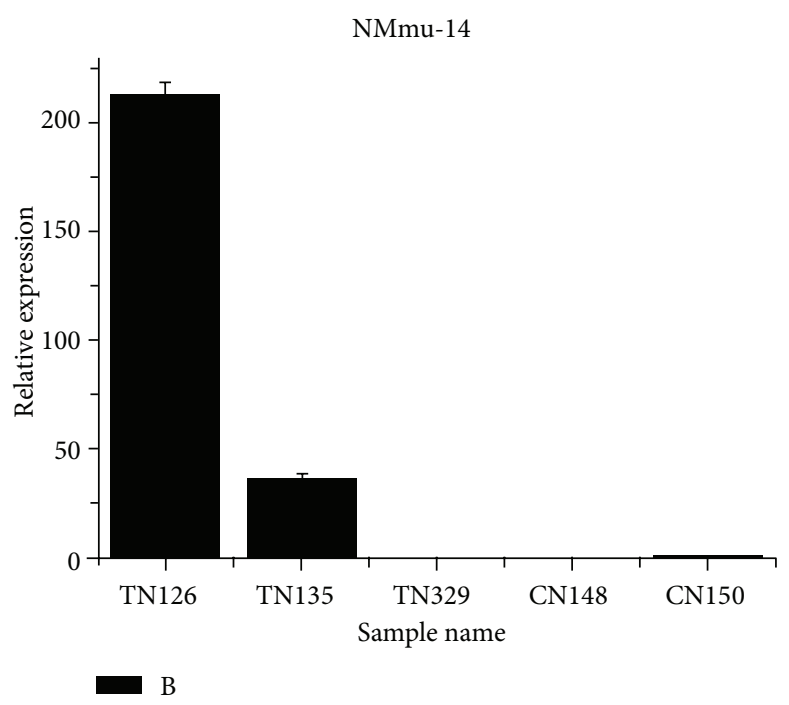

(i)

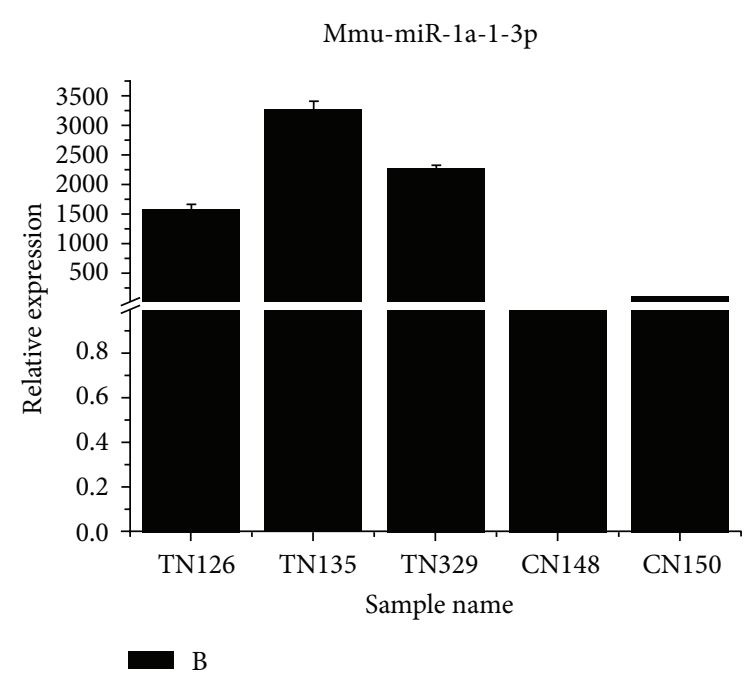

(h)

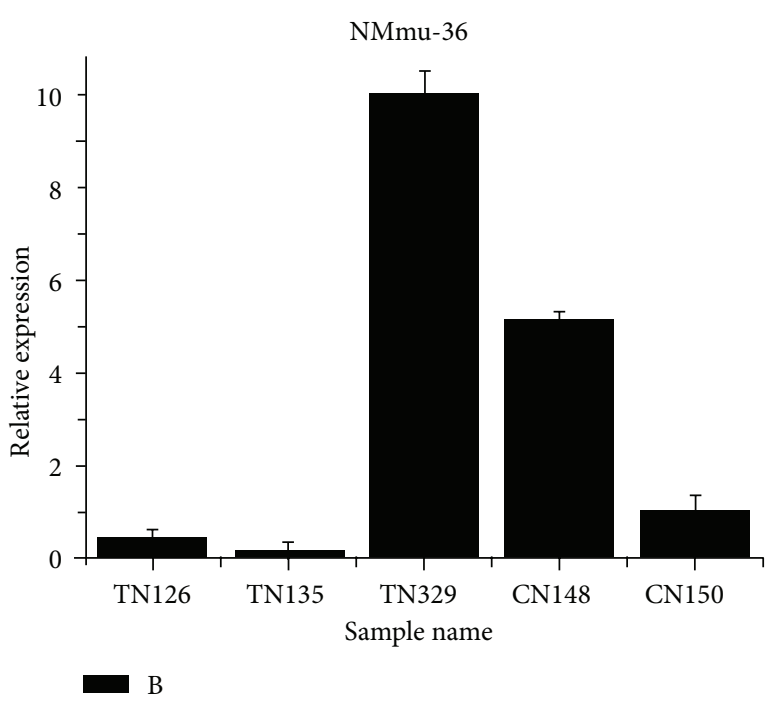

(j)

FIGURE 6: Validation of differentially expressed known and novel miRNAs: (a)-(j) eight differentially expressed and two novel miRNAs were selected for sequencing results validation.

indicated their important roles in the hypertrophic skeletal muscle of the myostatin propeptide transgenic mice.

\subsection{Differentially Expressed miRNA Responsible for Skeletal} Muscle Hypertrophy. A total of 57 differentially expressed miRNAs were identified; out of these miR-133a, miR-378a, and miR-26 were highly abundant. MiR-133a [23, 38], miR378a [50], miR-26a [51], miR-27b [52, 53], miR-21a [56], miR-29a [44], miR-148 [58], and miR-103 are skeletal muscle specific miRNAs and play a vital role in muscle differentiation and proliferation as reported in previous studies. miR-181 is upregulated during myocyte differentiation and represses homeobox protein Hox-A1l, a repressor of muscle-cell differentiation, thereby allowing new muscle growth [81]. The expression of miR-133 (miR-133a, miR-133b), miR-1, and miR181 (miR-181a, miR-181b, and miR-181c) was profiled in muscle from patients affected by myotonic dystrophy typel and it was observed that they were specifically induced during myogenesis [82]. miR-148a promotes myogenic differentiation and downregulates ROCK1 gene at the translational level and plays a positive role in skeletal muscle development through the RhoA/ROCK pathway [58].

MiR-1 and miR-133a were proposed to contribute in muscle hypertrophy by the removal of their transcriptional inhibitory effect on growth factors such as IGF-1. Likewise, a regulatory feedback loop was demonstrated in vitro where IGF-1 downregulated miR-1 via the Akt/FoxO3a pathway [55]. It was shown that FoxO3a increased the levels of miR1 resulting in reduced IGF-1 protein levels. The results from this study suggest that a number of differentially expressed miRNAs that could exert novel functions in skeletal muscle development. We identified targets for 20 highly abundant 


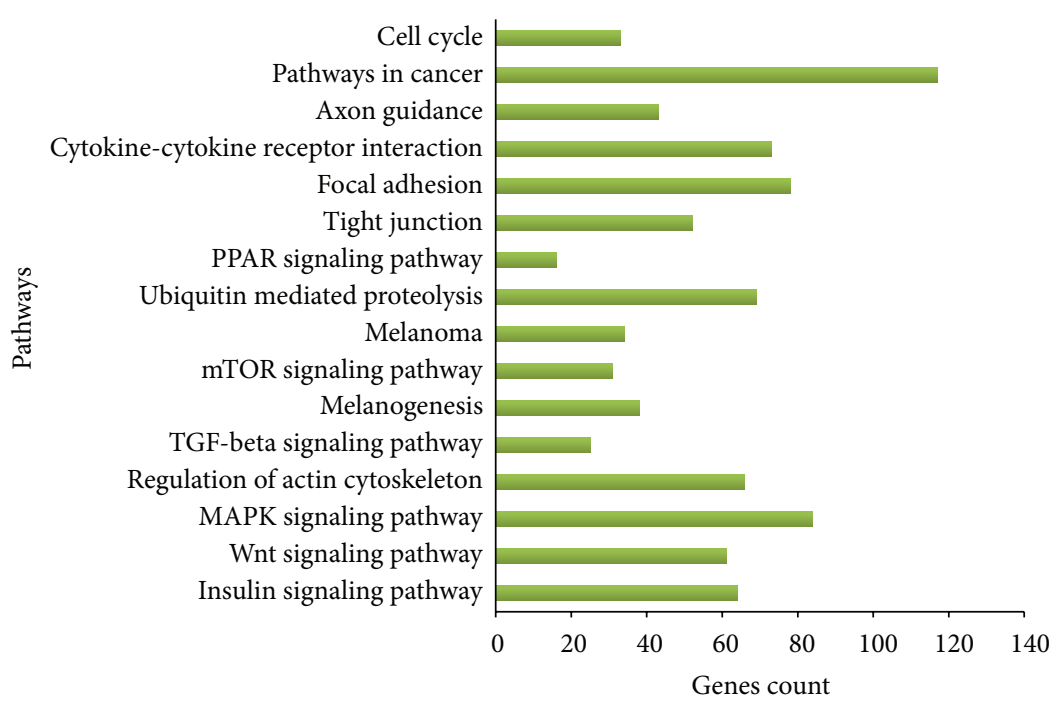

FIGURE 7: Pathways with respective gene numbers: top 16 Pathways predicted to be targeted differentially expressed miRNAs given on $Y$-axis and genes count on $X$-axis.

TABLE 4: Abundant differentially expressed miRNAs their targets and predicted pathways for targets.

\begin{tabular}{|c|c|c|c|c|}
\hline & miRNAs & MAPK signaling pathway & $\begin{array}{l}\text { TGF- } \beta \text { signaling } \\
\text { pathway }\end{array}$ & $\begin{array}{l}\text { mTOR signaling } \\
\text { Pathway }\end{array}$ \\
\hline 1 & miR-27b & $\begin{array}{c}\text { EGFR, RPS6KA5, KRAS, SOS1, } \\
\text { MAP2K4, MKNK2, CACNG2, FGF1, } \\
\text { RAPGEF2, CACNA1A, ACVR1C, PRKCB }\end{array}$ & $\begin{array}{l}\text { SMAD9, IFNG, RPS6KB1, } \\
\text { SMAD1, ACVR1C }\end{array}$ & $\begin{array}{l}\text { PDPK1, RPS6KB1, } \\
\text { RICTOR }\end{array}$ \\
\hline 2 & miR-16a & $\begin{array}{l}\text { CACNA2D1, FGF9, NF1, GNA12, } \\
\text { RAF1, MKNK1, MAPK8, AKT3 }\end{array}$ & & PIK3R1, AKT3 \\
\hline 3 & miR-21a & MAP3K7, NTF3, FASL, MAPK10, DUSP8 & PITX2 & \\
\hline 4 & $\mathrm{miR}-23 \mathrm{a}$ & $\begin{array}{l}\text { PTPN7, MAP4K4, MAP3K5, PAK2, } \\
\text { RRAS2, CACNA1E, FAS, STK4, CHUK }\end{array}$ & SMAD5, SMURF2 & PIK3CB, PIK3R3 \\
\hline 5 & miR-26a & RPS6KA6, RAP1A & & RPS6KA6, ULK1, ULK2 \\
\hline 6 & miR-29b & DUSP2, GNG12 & & VEGFA, IGF1, EIF4E2 \\
\hline 7 & miR-30b & TAOK1, RASA1, MAP3K12 & & PIK3CD, PRKAA2 \\
\hline 8 & $\operatorname{miR}-30 c$ & TAOK1, RASA1, MAP3K12 & & PIK3CD, PRKAA2 \\
\hline 9 & miR-30d & TAOK1, RASA1, MAP3K12 & & PIK3CD, PRKAA2 \\
\hline 10 & miR-30e & TAOK1, RASA1, MAP3K12 & & PIK3CD, PRKAA2 \\
\hline 11 & miR-101a & DUSP1, NLK, TGFBR1, CACNB2, FGF10 & TGFBR1, SMAD3 & HIF1A, PRKAA1 \\
\hline 12 & miR-103 & PRKCA, CDC25B & BMP2 & CAB39L, PGF \\
\hline 13 & $\operatorname{miR}-125 \mathrm{a}$ & TRAF6, MAP3K11 & INHBE & \\
\hline 14 & miR-133a & ARRB1, B230120H23RIK, FGF12, MECOM & PPP2CA, PPP2CB & \\
\hline 15 & miR-148b & SOS2, IKBKB, GADD45A & INHBB, NOG, ACVR1 & ULK3, RICTOR, MLST8 \\
\hline 16 & miR-199 & MAP3K4, ACVR1C & ACVR2A, RBL1, ACVR1C & MTOR \\
\hline 17 & miR-378a & $\begin{array}{l}\text { CACNG8, RASGRF1, ELK4, } \\
\text { PLA2G12A, TRAF6 }\end{array}$ & & \\
\hline 18 & $\operatorname{miR}-425 a$ & MEF2C, DUSP2, MAP2K1, TGFBR2, IL1A & TGFBR2, FST & IGF1, CAB39, FIGF \\
\hline 19 & miR-582 & BDNF, PPM1B, RASA2, TGFB2 & SMAD1, TGFB2 & \\
\hline 20 & miR-3068 & $\begin{array}{l}\text { RASGRP3, MAPT, FGF12, } \\
\text { CACNA1C, IL1A }\end{array}$ & PPP2CB, ID4, ACVR1 & PIK3CA \\
\hline
\end{tabular}




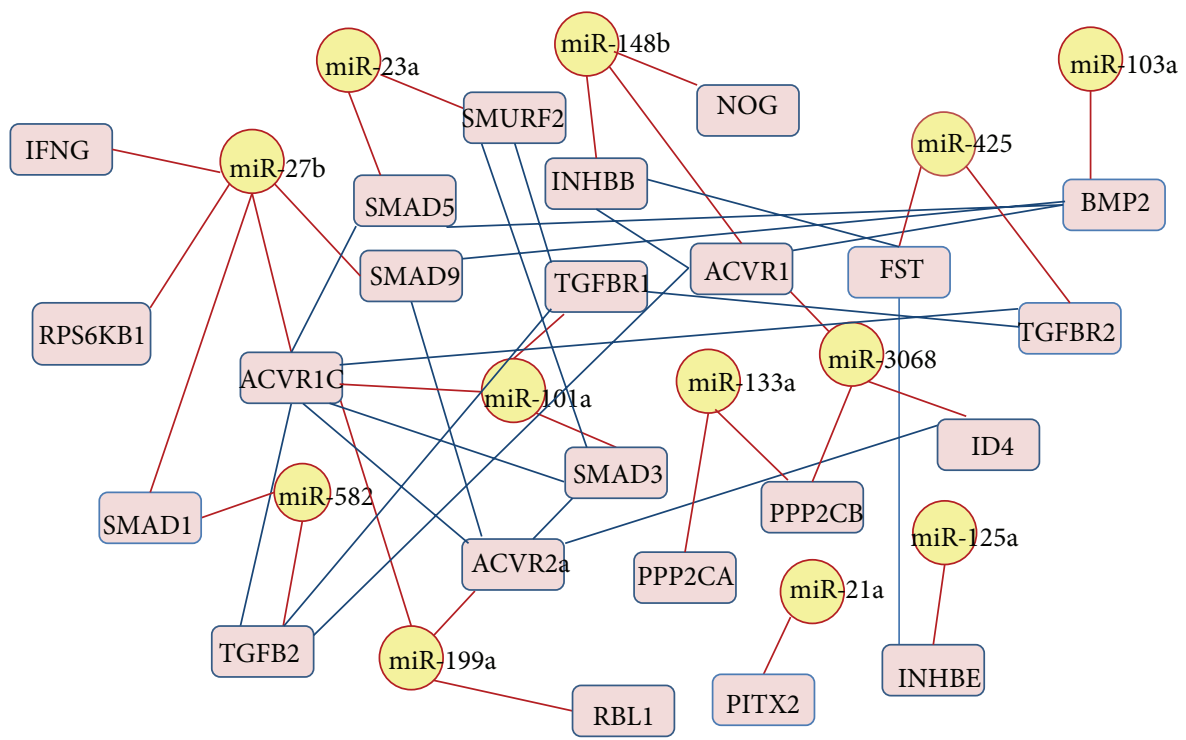

(a)

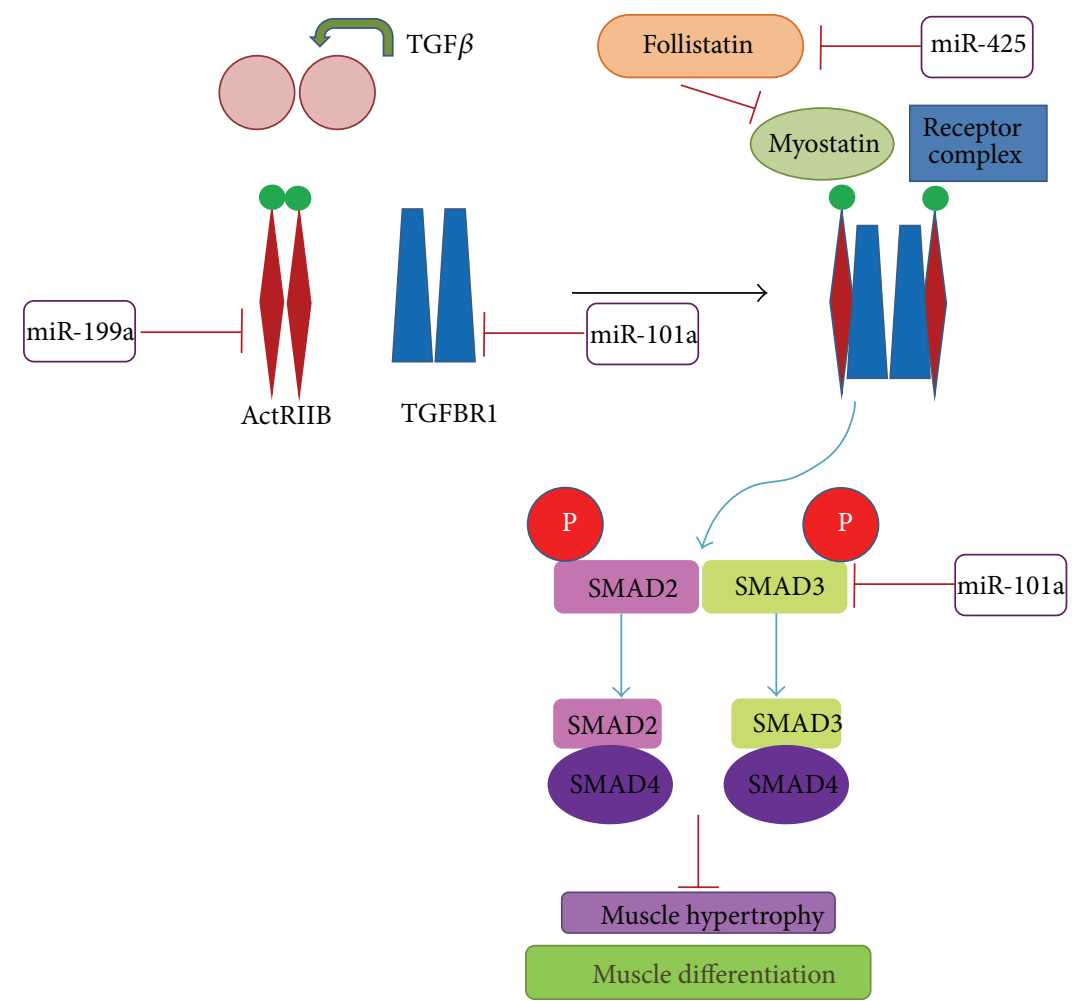

(b)

FIGURE 8: (a) Interaction network of differentially expressed miRNAs and their targets: network construction can be divided into two components: miRNA-target and target-target interaction, and candidate miRNA targets were predicted by mRDB. Target-target pairs interaction were searched from STRING database. In the network yellow nodes represented miRNAs and pink nodes represented targets, red lines denote miRNA-target interaction, and blue line denotes target-target interaction. These differentially expressed miRNAs predicted to be involved in TGFbeta signaling pathway. (b) MSTN signaling in skeletal muscle and miRNAs involved in the pathway: TGFb legend binds to the activin receptor two beta which interact with the TGFBR1 and they form a receptor complex, and mature myostatin inhibited by follistatin binds to the receptor complex which in turn phosphorylates regulatory SMADs, such as SMAD2 or 3. Phosphorylated regulatory SMADs are recognized by and associated with mediator co-SMADs, such as SMAD4, and translocated into the nucleus to interact with target gene promoter cis-elements and to regulate myostatin-specific gene expression. miRNAs were predicted to regulate the genes in TGFb signaling. 
differentially expressed miRNAs and predicted genes were analyzed to search the pathways. Sixteen pathways were selected including mTOR signaling, insulin-like growth factor, MAPK signaling and TGF- $\beta$ signaling and other pathways such as ubiquitin mediated proteolysis, focal adhesion, Wnt signaling pathway, regulation of actin cytoskeleton, cytokine-cytokine receptor interaction, tight junction, and cell cycle which are known to be involved in cell and tissue structure. Of these, we particularly focused on TGF- $\beta$ signaling, as many studies reported that TGF- $\beta$ family plays important roles in regulating muscle growth and development $[83,84]$. Pathways analysis predicted that miR-101a is targeting TGFBR1 and SMAD3, miR-582 is targeting TGFB2, SMAD1, miR-425 is targeting TGFBR2 and FST, miR-199a is targeting ACVR2a, miR-148 is targeting ACVR1, and miR-103 is targeting BMP2 in TGF- $\beta$ signaling pathway. A network was constructed to show the miRNA-target and target-target interaction in TGF- $\beta$ signaling (Figure 8(a)). Myostatin (MSTN) is predominantly expressed in skeletal muscle and plays a crucial role in muscle development and also signals through the TGF- $\beta$ branch [85] (Figures 8(a) and 8(b)). Myostatin binds to the TGFBR1 and makes a receptor complex for binding with ActRIIB, and activation of this receptor complex leads to the phosphorylation of SMAD2 and SMAD3.

\section{Conclusion}

This study was carried out to identify differentially expressed miRNA transcriptome in myostatin propeptide transgenic mice and to evaluate the miRNAs associated with hypertrophic skeletal muscle. Predicted target and pathway analysis concluded that miR-101a is targeting TGFBR1 and SMAD3, miR-425 is targeting TGFBR2 and FST, miR-582 is targeting SMAD1 and TGFB2, miR-148 is targeting ACVR1, and miR-199a is targeting AcvRla gene in the TGF- $\beta$ signaling pathway. As far as we have known, these miRNAs and their gene targets, which are crucial to myostatin signaling pathway, have not been reported earlier. Annotations of these major miRNAs to the myostatin signal pathway in relation to skeletal muscle hypertrophy can provide important guidance for the application of miRNA approaches to enhancing skeletal muscle growth and development.

\section{Conflict of Interests}

The authors declare that there is no conflict of interests regarding the publication of this paper.

\section{Acknowledgments}

The authors thank Dr. Mukesh (WII, Dehradun) for reviewing the paper and thank Dr. Wei Wei (HAU, Wuhan) for her timely help and support. Thanks are due to Yuanxin Miao for assistances in RNA extraction. This work was supported by the Chinese National Major Special Project on New Varieties Cultivation for Transgenic Organisms (2013ZX08006005), the Fundamental Research Funds for the Central
Universities of China, National Outstanding Youth Foundation of the National Natural Science Foundation of China (31025026), and Alan M Krassner and Ingeborg v. F. McKee Fund of the Hawaii Community Foundation (ID no. 13P60310).

\section{References}

[1] E. Zacharewicz, S. Lamon, and A. P. Russell, "MicroRNAs in skeletal muscle and their regulation with exercise, ageing, and disease," Frontiers in Physiology, vol. 4, no. 266, pp. 1-10, 2013.

[2] A. C. McPherron, A. M. Lawler, and S. Lee, "Regulation of skeletal muscle mass in mice by a new TGF- $\beta$ superfamily member," Nature, vol. 387, no. 6628, pp. 83-90, 1997.

[3] J. Jiao, T. Yuan, Y. Zhou et al., "Analysis of myostatin and its related factors in various porcine tissues," Journal of Animal Science, vol. 89, no. 10, pp. 3099-3106, 2011.

[4] A. Rebbapragada, H. Benchabane, J. L. Wrana, A. J. Celeste, and L. Attisano, "Myostatin signals through a transforming growth factor $\beta$-like signaling pathway to block adipogenesis," Molecular and Cellular Biology, vol. 23, no. 20, pp. 7230-7242, 2003.

[5] S. J. Lee, L. A. Reed, M. V. Davies et al., "Regulation of muscle growth by multiple ligands signaling through activin type II receptors," Proceedings of the National Academy of Sciences of the United States of America, vol. 102, no. 50, pp. 18117-18122, 2005.

[6] R. Scott Thies, T. Chen, M. V. Davies et al., "GDF-8 propeptide binds to GDF-8 and antagonizes biological activity by inhibiting GDF-8 receptor binding," Growth Factors, vol. 18, no. 4, pp. 251259, 2001.

[7] S. Bogdanovich, T. O. B. Krag, E. R. Barton et al., "Functional improvement of dystrophic muscle by myostatin blockade," Nature, vol. 420, no. 6914, pp. 418-421, 2002.

[8] B. Langley, M. Thomas, A. Bishop, M. Sharma, S. Gilmour, and R. Kambadur, "Myostatin inhibits myoblast differentiation by down-regulating MyoD expression," Journal of Biological Chemistry, vol. 277, no. 51, pp. 49831-49840, 2002.

[9] X. Zhu, S. Topouzis, L. F. Liang, and R. L. Stotish, "Myostatin signaling through $S m a d 2, S m a d 3$ and $S m a d 4$ is regulated by the inhibitory Smad7 by a negative feedback mechanism," Cytokine, vol. 26, no. 6, pp. 262-272, 2004.

[10] S. J. Lee, "Regulation of muscle mass by myostatin," Annual Review of Cell and Developmental Biology, vol. 20, pp. 61-86, 2004.

[11] J. Yang, T. Ratovitski, J. P. Brady, M. B. Solomon, K. D. Wells, and R. J. Wall, "Expression of myostatin pro domain results in muscular transgenic mice," Molecular Reproduction and Development, vol. 60, no. 3, pp. 351-361, 2001.

[12] J. Yang and B. Zhao, "Postnatal expression of myostatin propeptide cDNA maintained high muscle growth and normal adipose tissue mass in transgenic mice fed a high-fat diet," Molecular Reproduction and Development, vol. 73, no. 4, pp. 462-469, 2006.

[13] B. Zhao, E. J. Li, R. J. Wall, and J. Yang, "Coordinated patterns of gene expressions for adult muscle build-up in transgenic mice expressing myostatin propeptide," BMC Genomics, vol. 10, article 305, 2009.

[14] J. Yang, "Enhanced skeletal muscle for effective glucose homeostasis," Progress in Molecular Biology and Translational Science, vol. 121, pp. 133-163, 2014. 
[15] D. P. Bartel, "MicroRNAs: genomics, biogenesis, mechanism, and function," Cell, vol. 116, no. 2, pp. 281-297, 2004.

[16] W. Filipowicz, S. N. Bhattacharyya, and N. Sonenberg, "Mechanisms of post-transcriptional regulation by microRNAs: are the answers in sight?" Nature Reviews Genetics, vol. 9, no. 2, pp. 102114, 2008.

[17] J. Chen, T. E. Callis, and D. Wang, "microRNAs and muscle disorders," Journal of Cell Science, vol. 122, no. 1, pp. 13-20, 2009.

[18] J. Brennecke, A. Stark, R. B. Russell, and S. M. Cohen, "Principles of microRNA-target recognition," PLoS Biology, vol. 3, no. 3, p. e85, 2005.

[19] D. Grün, Y. Wang, D. Langenberger, K. C. Gunsalus, and N. Rajewsky, "MicroRNA target predictions across seven drosophilo species and comparison to mammalian targets," PLoS Computational Biology, vol. 1, no. 1, article e13, 2005.

[20] A. Krek, D. Grün, M. N. Poy et al., "Combinatorial microRNA target predictions," Nature Genetics, vol. 37, no. 5, pp. 495-500, 2005.

[21] B. P. Lewis, C. B. Burge, and D. P. Bartel, "Conserved seed pairing, often flanked by adenosines, indicates that thousands of human genes are microRNA targets," Cell, vol. 120, no. 1, pp. 15-20, 2005.

[22] X. Xie, J. Lu, E. J. Kulbokas et al., "Systematic discovery of regulatory motifs in human promoters and 3/ UTRs by comparison of several mammals," Nature, vol. 434, no. 7031, pp. 338345, 2005.

[23] J. F. Chen, E. M. Mandel, J. M. Thomson et al., "The role of microRNA-1 and microRNA-133 in skeletal muscle proliferation and differentiation," Nature Genetics, vol. 38, no. 2, pp. 228-233, 2006.

[24] M. Lagos-Quintana, R. Rauhut, A. Yalcin, J. Meyer, W. Lendeckel, and T. Tuschl, "Identification of tissue-specific MicroRNAs from mouse," Current Biology, vol. 12, no. 9, pp. 735$739,2002$.

[25] E. J. Lee, M. Baek, Y. Gusev, D. J. Brackett, G. J. Nuovo, and T. D. Schmittgen, "Systematic evaluation of microRNA processing patterns in tissues, cell lines, and tumors," $R N A$, vol. 14, no. 1, pp. 35-42, 2008.

[26] T. G. McDaneld, T. P. L. Smith, M. E. Doumit et al., "MicroRNA transcriptome profiles during swine skeletal muscle development," BMC Genomics, vol. 10, article 77, 2009.

[27] N. Sdassi, L. Silveri, J. Laubier et al., "Identification and characterization of new miRNAs cloned from normal mouse mammary gland," BMC Genomics, vol. 10, article 149, 2009.

[28] L. F. Sempere, S. Freemantle, I. Pitha-Rowe, E. Moss, E. Dmitrovsky, and V. Ambros, "Expression profiling of mammalian microRNAs uncovers a subset of brain-expressed microRNAs with possible roles in murine and human neuronal differentiation," Genome Biology, vol. 5, no. 3, article R13, 2004.

[29] A. A. Aboobaker, P. Tomancak, N. Patel, G. M. Rubin, and E. C. Lai, "Drosophila microRNAs exhibit diverse spatial expression patterns during embryonic development," Proceedings of the National Academy of Sciences of the United States of America, vol. 102, no. 50, pp. 18017-18022, 2005.

[30] J. R. O'Rourke, S. A. Georges, H. R. Seay et al., "Essential role for Dicer during skeletal muscle development," Developmental Biology, vol. 311, no. 2, pp. 359-368, 2007.

[31] N. Liu, A. H. Williams, J. M. Maxeiner et al., "MicroRNA-206 promotes skeletal muscle regeneration and delays progression of Duchenne muscular dystrophy in mice," Journal of Clinical Investigation, vol. 122, no. 6, pp. 2054-2065, 2012.
[32] A. H. Williams, N. Liu, E. van Rooij, and E. N. Olson, "MicroRNA control of muscle development and disease," Current Opinion in Cell Biology, vol. 21, no. 3, pp. 461-469, 2009.

[33] E. M. Small, J. R. O'Rourke, V. Moresi et al., "Regulation of PI3-kinase/Akt signaling by muscle-enriched microRNA-486," Proceedings of the National Academy of Sciences of the United States of America, vol. 107, no. 9, pp. 4218-4223, 2010.

[34] E. van Rooij, N. Liu, and E. N. Olson, "MicroRNAs flex their muscles," Trends in Genetics, vol. 24, no. 4, pp. 159-166, 2008.

[35] E. van Rooij, D. Quiat, B. A. Johnson et al., "A family of microRNAs encoded by myosin genes governs myosin expression and muscle performance," Developmental Cell, vol. 17, no. 5, pp. 662673, 2009.

[36] J. J. McCarthy and K. A. Esser, "MicroRNA-1 and microRNA133a expression are decreased during skeletal muscle hypertrophy," Journal of Applied Physiology, vol. 102, no. 1, pp. 306-313, 2007.

[37] T. E. Callis, Z. Deng, J. F. Chen, and D. Z. Wang, "Muscling through the microRNA world," Experimental Biology and Medicine, vol. 233, no. 2, pp. 131-138, 2008.

[38] Y. Feng, L. L. Niu, W. Wei et al., "A feedback circuit between miR-133 and the ERK1/2 pathway involving an exquisite mechanism for regulating myoblast proliferation and differentiation," Cell Death and Disease, vol. 4, no. 11, article e934, 2013.

[39] N. Liu and E. N. Olson, "MicroRNA regulatory networks in cardiovascular development," Developmental Cell, vol. 18, no. 4, pp. 510-525, 2010.

[40] P. K. Rao, R. M. Kumar, M. Farkhondeh, S. Baskerville, and H. F. Lodish, "Myogenic factors that regulate expression of musclespecific microRNAs," Proceedings of the National Academy of Sciences of the United States of America, vol. 103, no. 23, pp. 87218726, 2006.

[41] M. I. Rosenberg, S. A. Georges, A. Asawachaicharn, E. Analau, and S. J. Tapscott, "MyoD inhibits Fstll and Utrn expression by inducing transcription of miR-206," Journal of Cell Biology, vol. 175, no. 1, pp. 77-85, 2006.

[42] N. Liu, A. H. Williams, Y. Kim et al., "An intragenic MEF2dependent enhancer directs muscle-specific expression of microRNAs 1 and 133," Proceedings of the National Academy of Sciences of the United States of America, vol. 104, no. 52, pp. 20844-20849, 2007.

[43] H. K. Kim, Y. S. Lee, U. Sivaprasad, A. Malhotra, and A. Dutta, "Muscle-specific microRNA miR-206 promotes muscle differentiation," Journal of Cell Biology, vol. 174, no. 5, pp. 677687, 2006.

[44] W. Wei, H.-B. He, W.-Y. Zhang et al., "MiR-29 targets Akt3 to reduce proliferation and facilitate differentiation of myoblasts in skeletal muscle development," Cell Death and Disease, vol. 4, article e668, no. 6, 2013.

[45] S. Rachagani, Y. Cheng, and J. M. Reecy, "Myostatin genotype regulates muscle-specific miRNA expression in mouse pectoralis muscle," BMC Research Notes, vol. 3, article 297, 2010.

[46] S. Miretti, E. Martignani, P. Accornero, and M. Baratta, "Functional effect of mir-27b on myostatin expression: a relationship in piedmontese cattle with double-muscled phenotype," BMC Genomics, vol. 14, no. 1, article 194, 2013.

[47] C. McFarlane, A. Vajjala, H. Arigela et al., "Negative autoregulation of myostatin expression is mediated by $\mathrm{Smad} 3$ and microRNA-27," PLoS ONE, vol. 9, no. 1, Article ID e87687, 2014.

[48] T. Li, R. Wu, Y. Zhang, and D. Zhu, "A systematic analysis of the skeletal muscle miRNA transcriptome of chicken varieties 
with divergent skeletal muscle growth identifies novel miRNAs and differentially expressed miRNAs," BMC Genomics, vol. 12, article 186, 2011.

[49] B. K. Dey, J. Gagan, and A. Dutta, "miR-206 and -486 induce myoblast differentiation by downregulating Pax7," Molecular and Cellular Biology, vol. 31, no. 1, pp. 203-214, 2011.

[50] J. Gagan, B. K. Dey, R. Layer, Z. Yan, and A. Dutta, "MicroRNA378 targets the myogenic repressor MyoR during myoblast differentiation," Journal of Biological Chemistry, vol. 286, no. 22, pp. 19431-19438, 2011.

[51] B. K. Dey, J. Gagan, Z. Yan, and A. Dutta, "miR-26a is required for skeletal muscle differentiation and regeneration in mice," Genes and Development, vol. 26, no. 19, pp. 2180-2191, 2012.

[52] C. G. Crist, D. Montarras, G. Pallafacchina et al., "Muscle stem cell behavior is modified by microRNA-27 regulation of Pax3 expression," Proceedings of the National Academy of Sciences of the United States of America, vol. 106, no. 32, pp. 13383-13387, 2009.

[53] D. L. Allen and A. S. Loh, "Posttranscriptional mechanisms involving microRNA-27a and b contribute to fast-specific and glucocorticoid-mediated myostatin expression in skeletal muscle," American Journal of Physiology: Cell Physiology, vol. 300, no. 1, pp. C124-C137, 2011.

[54] M. Nielsen, J. H. Hansen, J. Hedegaard et al., "MicroRNA identity and abundance in porcine skeletal muscles determined by deep sequencing," Animal Genetics, vol. 41, no. 2, pp. 159-168, 2010.

[55] L. Elia, R. Contu, M. Quintavalle et al., "Reciprocal regulation of microrna-1 and insulin-like growth factor-1 signal transduction cascade in cardiac and skeletal muscle in physiological and pathological conditions," Circulation, vol. 120, no. 23, pp. 2377$2385,2009$.

[56] K. H. Haider, N. M. Idris, H. W. Kim, R. P. H. Ahmed, J. Shujia, and M. Ashraf, "MicroRNA-21 is a key determinant in IL$11 /$ Stat 3 anti-apoptotic signalling pathway in preconditioning of skeletal myoblasts," Cardiovascular Research, vol. 88, no. 1, pp. 168-178, 2010.

[57] Y. Ge, Y. Sun, and J. Chen, "IGF-II is regulated by microRNA125b in skeletal myogenesis," Journal of Cell Biology, vol. 192, no. 1, pp. 69-81, 2011.

[58] J. Zhang, Z. Ying, Z. Tang, L. Long, and K. Li, "MicroRNA-148a promotes myogenic differentiation by targeting the ROCK1 gene," The Journal of Biological Chemistry, vol. 287, no. 25, pp. 21093-21101, 2012.

[59] H. J. Fu, J. Zhu, M. Yang et al., "A novel method to monitor the expression of microRNAs," Molecular Biotechnology, vol. 32, no. 3, pp. 197-204, 2006.

[60] K. Strimmer, "fdrtool: a versatile R package for estimating local and tail area-based false discovery rates," Bioinformatics, vol. 24, no. 12, pp. 1461-1462, 2008.

[61] M. R. Friedländer, W. Chen, C. Adamidi et al., "Discovering microRNAs from deep sequencing data using miRDeep," Nature Biotechnology, vol. 26, no. 4, pp. 407-415, 2008.

[62] I. L. Hofacker, "Vienna RNA secondary structure server," Nucleic Acids Research, vol. 31, no. 13, pp. 3429-3431, 2003.

[63] X. Wang, "miRDB: a microRNA target prediction and functional annotation database with a wiki interface," RNA, vol. 14, no. 6, pp. 1012-1017, 2008.

[64] H. Dweep, C. Sticht, P. Pandey, and N. Gretz, "MiRWalkdatabase: prediction of possible miRNA binding sites by "walking" the genes of three genomes," Journal of Biomedical Informatics, vol. 44, no. 5, pp. 839-847, 2011.
[65] D. W. Huang, B. T. Sherman, and R. A. Lempicki, "Systematic and integrative analysis of large gene lists using DAVID bioinformatics resources," Nature Protocols, vol. 4, no. 1, pp. 44-57, 2009.

[66] D. W. Huang, B. T. Sherman, and R. A. Lempicki, "Bioinformatics enrichment tools: paths toward the comprehensive functional analysis of large gene lists," Nucleic Acids Research, vol. 37, no. 1, pp. 1-13, 2009.

[67] P. Nelson, M. Kiriakidou, A. Sharma, E. Maniataki, and Z. Mourelatos, "The microRNA world: small is mighty," Trends in Biochemical Sciences, vol. 28, no. 10, pp. 534-540, 2003.

[68] F. Kuchenbauer, R. D. Morin, B. Argiropoulos et al., "In-depth characterization of the microRNA transcriptome in a leukemia progression model," Genome Research, vol. 18, no. 11, pp. 17871797, 2008.

[69] L. Pantano, X. Estivill, and E. Martí, "SeqBuster, a bioinformatic tool for the processing and analysis of small RNAs datasets, reveals ubiquitous miRNA modifications in human embryonic cells," Nucleic Acids Research, vol. 38, no. 5, p. e34, 2009.

[70] L. Jia, Y. F. Li, G. F. Wu et al., "MiRNA-199a-3p regulates C2C12 myoblast differentiation through IGF-1/AKT/mTOR signal pathway," International Journal of Molecular Science, vol. 15, no. 1, pp. 296-308, 2014.

[71] C. Vaz, H. M. Ahmad, P. Sharma et al., "Analysis of microRNA transcriptome by deep sequencing of small RNA libraries of peripheral blood," BMC Genomics, vol. 11, no. 1, article 288, 2010.

[72] A. Alteri, F. de Vito, G. Messina et al., "Cyclin D1 is a major target of miR-206 in cell differentiation and transformation," Cell Cycle, vol. 12, no. 24, pp. 3781-3790, 2013.

[73] K. Goljanek-Whysall, H. Pais, T. Rathjen, D. Sweetman, T. Dalmay, and A. Münsterberg, "Regulation of multiple target genes by miR-1 and miR-206 is pivotal for $\mathrm{C} 2 \mathrm{C} 12$ myoblast differentiation," Journal of Cell Science, vol. 125, part 15, pp. 3590-3600, 2012.

[74] C. Y. Lin, H. C. Lee, C. Y. Fu et al., "miR-1 and miR-206 target different genes to have opposing roles during angiogenesis in zebrafish embryos," Nature Communication, vol. 4, no. 2829, 2013.

[75] C. Stahlhut, Y. Suárez, J. Lu, Y. Mishima, and A. J. Giraldez, "miR-1 and miR-206 regulate angiogenesis by modulating VegfA expression in zebrafish," Development, vol. 139, no. 23, pp. 4356-4364, 2012.

[76] Z. Deng, J. F. Chen, and D. Z. Wang, “Transgenic overexpression of miR-133a in skeletal muscle," BMC Musculoskeletal Disorders, vol. 12, article 115, 2011.

[77] D. Sweetman, K. Goljanek, T. Rathjen et al., "Specific requirements of MRFs for the expression of muscle specific microRNAs, miR-1, miR-206 and miR-133," Developmental Biology, vol. 321, no. 2, pp. 491-499, 2008.

[78] N. Nakajima, T. Takahashi, R. Kitamura et al., "MicroRNA1 facilitates skeletal myogenic differentiation without affecting osteoblastic and adipogenic differentiation," Biochemical and Biophysical Research Communications, vol. 350, no. 4, pp. 10061012, 2006.

[79] C. Xu, Y. Lu, Z. Pan et al., "The muscle-specific microRNAs miR-1 and miR-133 produce opposing effects on apoptosis by targeting HSP60, HSP70 and caspase-9 in cardiomyocytes," Journal of Cell Science, vol. 120, part 17, pp. 3045-3052, 2007.

[80] I. Güller and A. P. Russell, "MicroRNAs in skeletal muscle: Their role and regulation in development, disease and function," Journal of Physiology, vol. 588, no. 21, pp. 4075-4087, 2010. 
[81] I. Naguibneva, M. Ameyar-Zazoua, A. Polesskaya et al., "The microRNA miR-181 targets the homeobox protein Hox-A1l during mammalian myoblast differentiation," Nature Cell Biology, vol. 8, no. 3, pp. 278-284, 2006.

[82] S. Gambardella, F. Rinaldi, S. M. Lepore et al., "Overexpression of microRNA-206 in the skeletal muscle from myotonic dystrophy type 1 patients," Journal of Translational Medicine, vol. 8, article 48, 2010.

[83] H. D. Kollias and J. C. McDermott, "Transforming growth factor- $\beta$ and myostatin signaling in skeletal muscle," Journal of Applied Physiology, vol. 104, no. 3, pp. 579-587, 2008.

[84] T. N. Burks and R. D. Cohn, "Role of TGF- $\beta$ signaling in inherited and acquired myopathies," Skeletal Muscle, vol. 1, no. 1, article 19, 2011.

[85] A. Moustakas and C. Heldin, "Non-Smad TGF- $\beta$ signals," Journal of Cell Science, vol. 118, no. 16, pp. 3573-3584, 2005. 

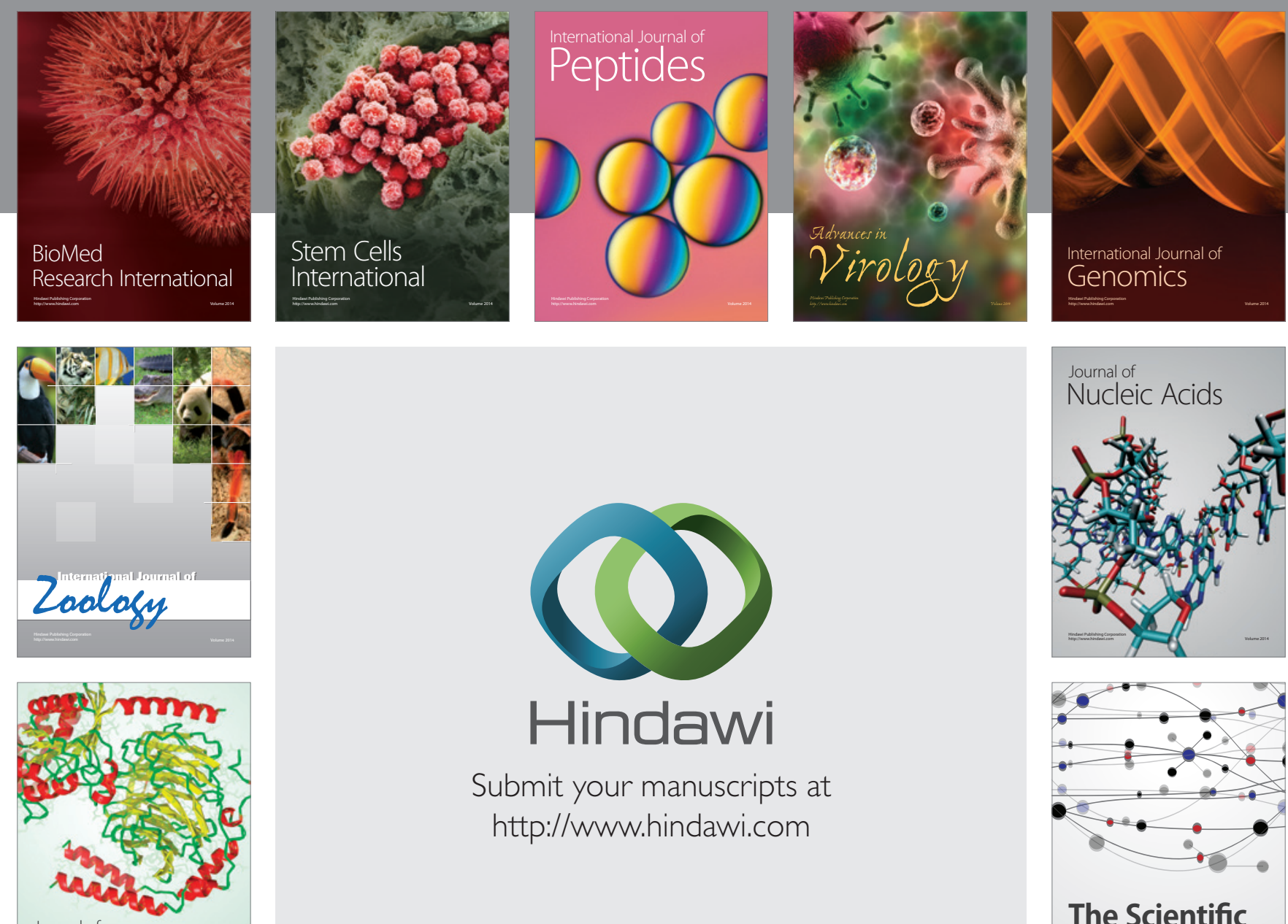

Submit your manuscripts at

http://www.hindawi.com

Journal of
Signal Transduction
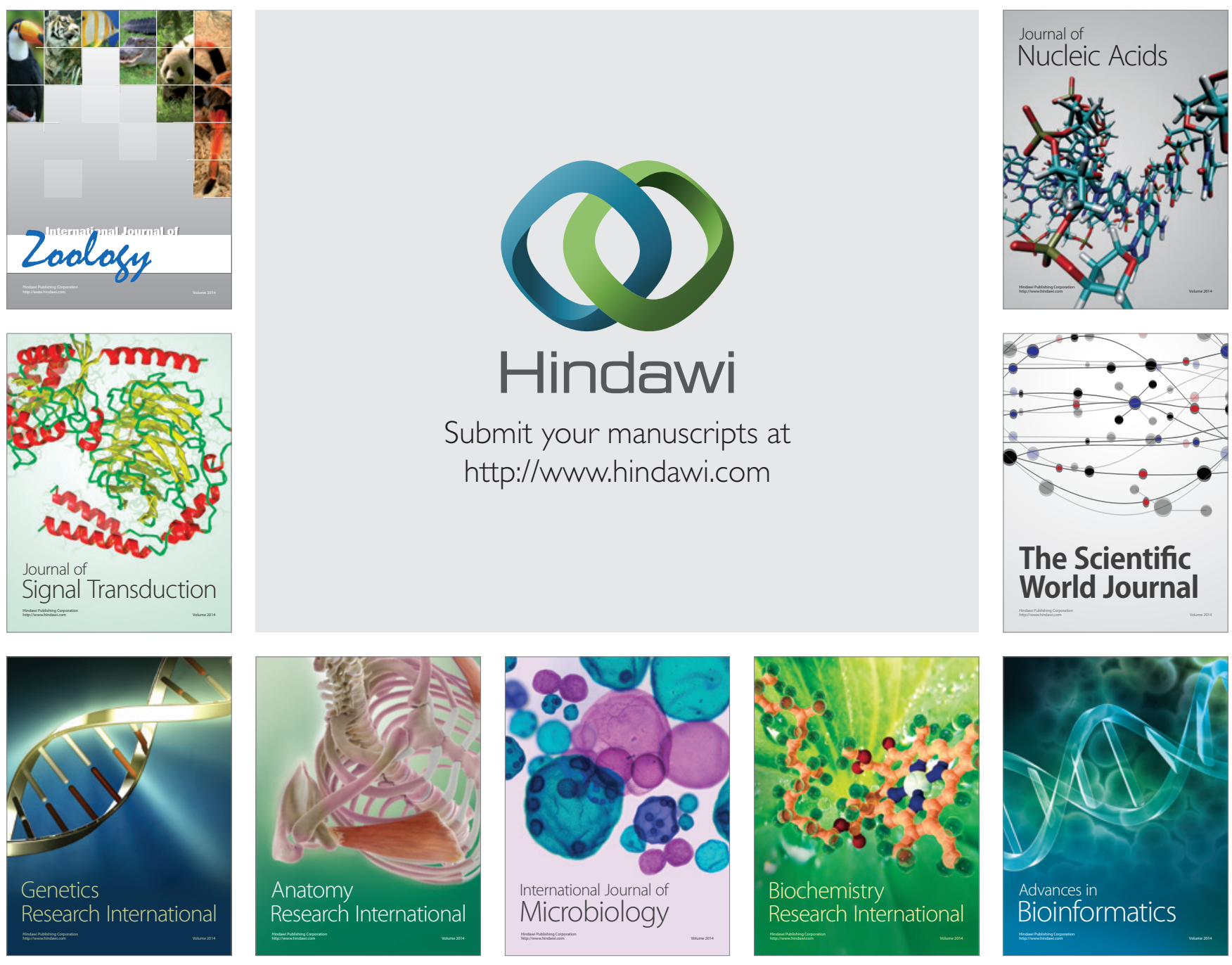

The Scientific World Journal
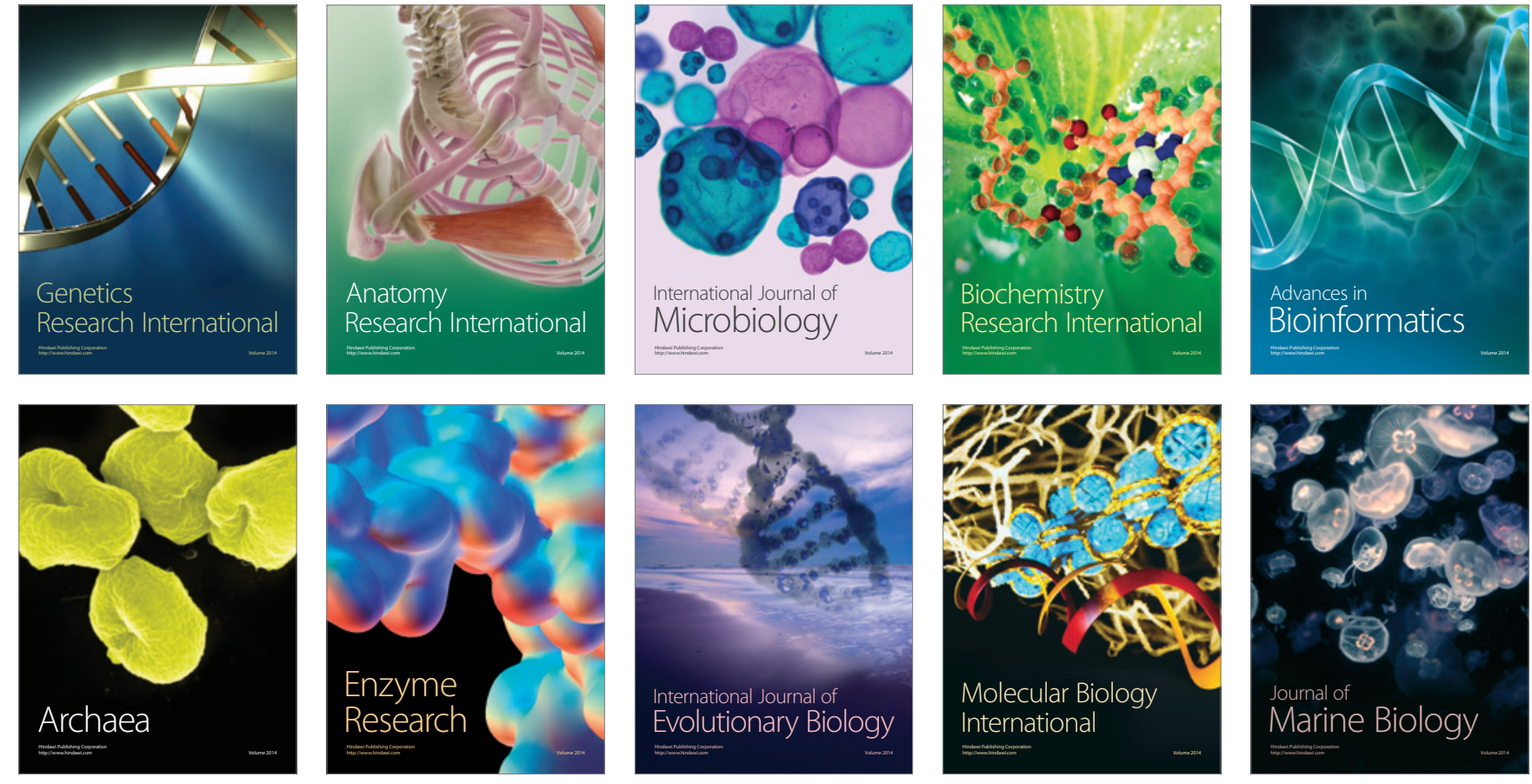